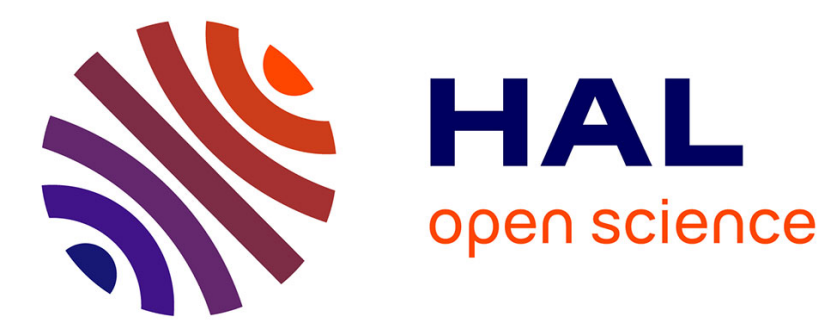

\title{
Viscous effects in the absolute-convective instability of the Batchelor vortex
}

\author{
C. Olendraru, Antoine Sellier
}

\section{To cite this version:}

C. Olendraru, Antoine Sellier. Viscous effects in the absolute-convective instability of the Batchelor vortex. Journal of Fluid Mechanics, 2002, 459 (may), pp.371-396. 10.1017/s0022112002008029 . hal-01024918

\section{HAL Id: hal-01024918 \\ https://hal-polytechnique.archives-ouvertes.fr/hal-01024918}

Submitted on 11 Sep 2014

HAL is a multi-disciplinary open access archive for the deposit and dissemination of scientific research documents, whether they are published or not. The documents may come from teaching and research institutions in France or abroad, or from public or private research centers.
L'archive ouverte pluridisciplinaire HAL, est destinée au dépôt et à la diffusion de documents scientifiques de niveau recherche, publiés ou non, émanant des établissements d'enseignement et de recherche français ou étrangers, des laboratoires publics ou privés. 


\title{
Viscous effects in the absolute-convective instability of the Batchelor vortex
}

\author{
By C. OLENDRARU AND A. SELLIER \\ Laboratoire d'Hydrodynamique (LadHyX), CNRS-Ecole Polytechnique, F-91128 Palaiseau, France
}

(Received 10 December 1998 and in revised form 30 November 2001)

The effects of viscosity on the instability properties of the Batchelor vortex are investigated. The characteristics of spatially amplified branches are first documented in the convectively unstable regime for different values of the swirl parameter $q$ and the co-flow parameter $a$ at several Reynolds numbers $R e$. The absolute-convective instability transition curves, determined by the Briggs-Bers zero-group velocity criterion, are delineated in the $(a, q)$-parameter plane as a function of $R e$. The azimuthal wavenumber $m$ of the critical transitional mode is found to depend on the magnitude of the swirl $q$ and on the jet $(a>-0.5)$ or wake $(a<-0.5)$ nature of the axial flow. At large Reynolds numbers, the inviscid results of Olendraru et al. (1999) are recovered. As the Reynolds number decreases, the pocket of absolute instability in the $(a, q)$-plane is found to shrink gradually. At $R e=667$, the critical transitional modes for swirling jets are $m=-2$ or $m=-3$ and absolute instability prevails at moderate swirl values even in the absence of counterflow. For higher swirl levels, the bending mode $m=-1$ becomes critical. The results are in good overall agreement with those obtained by Delbende et al. (1998) at the same Reynolds number. However, a bending $(m=+1)$ viscous mode is found to partake in the outer absolute-convective instability transition for jets at very low positive levels of swirl. This asymmetric branch is the spatial counterpart of the temporal viscous mode isolated by Khorrami (1991) and Mayer \& Powell (1992). At $R e=100$, the critical transitional mode for swirling jets is $m=-2$ at moderate and high swirl values and, in order to trigger an absolute instability, a slight counterflow is always required. A bending $(m=+1)$ viscous mode again becomes critical at very low swirl values. For wakes $(a<-0.5)$ the critical transitional mode is always found to be the bending mode $m=-1$, whatever the Reynolds number. However, above $q=1.5$, near-neutral centre modes are found to define a tongue of weak absolute instability in the $(a, q)$-plane. Such modes had been analytically predicted by Stewartson \& Brown (1985) in a strictly temporal inviscid framework.

\section{Introduction}

Considerable efforts have been directed toward understanding the stability characteristics of vortices with axial flow. Depending both on the amount of swirl and on the outer uniform axial velocity, such flows may be highly sensitive to perturbations. In many technical applications it can even result in breakdown, i.e. in sudden changes in the structure of the vortex core. Among other examples, we should cite the slowly evolving leading-edge vortices on a delta wing and confined vortices in pipes. To the present day, the instability properties have been mainly investigated within the 
framework of a linear analysis (for a comprehensive review of vortex stability see Ash \& Khorrami 1995).

The present study takes as mean-velocity profile the familiar Batchelor (1964) vortex since it both satisfactorily approximates slowly evolving trailing vortices (Faler \& Leibovich 1977; Garg \& Leibovich 1979) and experimental velocity profiles for vane-guide-generated pipe vortices (Leibovich 1983), except at very low Reynolds numbers where wall effects become significant. Only two non-dimensional control parameters describe this axisymmetric and parallel rotating flow: the swirl ratio $q$ which measures the relative importance of the swirl velocity with respect to the axial velocity difference, and the axial co-flow parameter $a$ which measures the relative importance of the external axial velocity with respect to the axial velocity difference.

The strictly temporal (given real axial wavenumber $k$ and unknown complex frequency $\omega$ ) instability analysis of the Batchelor $(a, q)$-vortex has been extensively addressed both in the inviscid and in the fully viscous cases. In these investigations, one actually sets $a=0$ (by invoking a Galilean shift which only affects $\omega_{r}$ ) and look for the temporal modes $\omega(k ; m, q, R e)$ at different azimuthal wavenumbers $m$ and Reynolds numbers $R e$. For each setting $(k ; m, q, R e)$ several modes may arise: the 'primary' mode (i.e. the most unstable eigenvalue $\omega$ ) and higher less-amplified modes.

In the inviscid case and at $q=0$, only the bending modes $m= \pm 1$ are unstable, i.e. of positive growth rate $\omega_{i}$ (Lessen \& Singh 1974). The influence of strictly positive swirl on each inviscid mode, denoted by $\omega(k ; m, q)$, was found to depend on its azimuthal wavenumber in the following manner (see Lessen, Singh \& Paillet 1974 for the 'primary' modes and Duck \& Foster 1980 and Mayer \& Powell 1992 for higher modes). For $m=0$ and $m \geqslant 2$, inviscid modes remain stable whatever the magnitude of the swirl. For $m=+1$, inviscid modes become unstable within the range $0 \leqslant q \leqslant q_{1} \sim 0.0739$. For $m \leqslant-1$, inviscid modes are highly unstable at moderate values of $q$ and they become damped as $q$ approaches 1.5. Furthermore, the growth rate of the $m \leqslant-1$ modes increases with $-m$. Finally, note that nearly neutral so-called centre modes have been analytically predicted by Stewartson \& Brown (1985) for moderate azimuthal wavenumbers in specific ranges of swirl above 1.5. As established by Leibovich \& Stewartson (1983) and later confirmed numerically by Mayer \& Powell (1992), the maximum growth rate admits a finite limit as $|m|$ tends to infinity and the associated eigenfunctions become strongly localized in the form of 'ring' modes. Moreover, Leibovich \& Stewartson (1983), Leibovich \& Stewartson (1983) and Stewartson \& Capell (1985) have shown that, for large values of $-m$, the 'primary' and higher inviscid modes tend to coalesce near neutrality. This behaviour results in numerical difficulties when computing marginally unstable modes. It is worth mentioning that other basic swirling jet/wake profiles have been examined recently from the point of view of their convective/absolute instability properties: the Rankine vortex with plug flow axial velocity profile (Loiseleux, Chomaz \& Huerre 1998) and the swirling jet/wake shear layer (Loiseleux, Delbende \& Huerre 2000).

Viscosity effects are generally believed to have, for unconfined flows, a stabilizing influence on 'inviscid' modes. For fine-scale perturbations, such as modes with large values of $-m$, this is indeed the case (Mayer \& Powell 1992); these modes $\omega(k ; m, q, R e)$ are stable below a critical Reynolds number $\operatorname{Re}_{c}(m)=O\left(m^{2}\right)$ and they quickly asymptote, for $\operatorname{Re} \gg \operatorname{Re}_{c}(m)$, to the corresponding inviscid mode $\omega(k ; m, q)$. For disturbances of low-to-medium azimuthal wavenumbers, the role of viscosity becomes less intuitively obvious; Lessen \& Paillet (1974) found a critical Reynolds number for the 'primary' modes at $m=-1, m=-2$ and $m=-3$ but Khorrami (1992) reports that, in a certain range of Reynolds numbers, viscosity destabilizes the bending mode 
$m=-1$ with mode-switching and mode-crossing phenomena involving 'primary' and higher bending modes $m=-1$. Moreover, Khorrami (1991) discovered both axisymmetric $(m=0)$ and bending $(m=1)$ centre viscous modes with growth rates several orders of magnitude lower than those of inviscid modes. These viscous modes only exist within a domain of the $(q, k)$-plane where inviscid instabilities do not occur. For $R e<100$, Mayer \& Powell (1992) also discovered higher and near-neutral viscous bending modes $m=1$ of small axial wavenumbers. At large Reynolds numbers, Duck \& Khorrami (1992) resorted to an asymptotic analysis to demonstrate that viscous modes become neutrally stable with $\omega_{i}=O\left(R e^{-1}\right)$.

It is tempting to compare the results of temporal stability analyses with experimental observations of the vortex breakdown phenomenon. This sudden transition between a jet-like and a wake-like rotating flow is associated with an expansion of the vortex core, as reviewed by Leibovich $(1978,1983)$ and Delery $(1990)$. The vortex exhibits within its viscous core a stagnation point which is followed by an intricate structure consisting of one or several regions of reverse axial flow. The experimental investigations of Garg \& Leibovich (1979) have shown that time-averaged velocity profiles at different stations, both upstream and downstream of this stagnation point, were satisfactorily approximated by Batchelor vortices, respectively, with $q>1.5$ (i.e. temporally stable) and $q<1.5$ (i.e. temporally unstable). The downstream flow was also found to promote bending disturbances $m= \pm 1$ only. However, the strictly temporal analysis fails to account for the occurrence of vortex breakdown. Following Benjamin (1962), we may also attempt to consider vortex breakdown as a transition between an incoming supercritical flow (i.e. which allows downstream propagating perturbations only) and a subcritical outgoing flow (i.e. which allows both upstream and downstream propagating disturbances). Originally developed for axisymmetric, non-dispersive and low-wavenumber disturbances by Benjamin (1962), this idea has been further extended to arbitrary temporal perturbations by Tsai \& Widnall (1980).

Supercritical or subcritical concepts appear somewhat analogous to the notions of convective or absolute instabilities, respectively. The latter distinction, which actually requires a fully spatio-temporal analysis, has been pioneered in plasma instabilities (Briggs 1964; Bers 1983) and more recently applied to fluid-dynamical instabilities in parallel and weakly spatially developing open flows (Huerre \& Monkewitz 1990; Huerre \& Rossi 1998; Huerre 2000). The nature of a given flow depends on the largetime asymptotic behaviour of the linear impulse response; the flow is convectively unstable if the amplified disturbances move away from the source and absolutely unstable when amplified perturbations invade the entire flow.

The absolute-convective nature of the instability is determined by applying the Briggs-Bers zero-group velocity criterion to the dispersion relation for fully complex $(k, \omega)$ pairs. In order to determine the transition from convective to absolute instability, it is sufficient to detect the occurrence of so-called pinch points in the characteristics of spatial instability waves (given complex frequency $\omega$ and unknown complex axial wavenumber $k$ ). This property motivates the present spatial instability analysis of the Batchelor $(a, q)$-vortex. Contrary to the temporal setting, it is not possible to restrict spatial investigations to the case $a=0$. In the inviscid case, Olendraru et al. $(1996,1999)$ have employed a shooting method to determine the spatial growth rates of the 'primary' helical modes $m= \pm 1, m=-2$ and $m=-3$ as a function of the swirl and co-flow parameters $q$ and $a$. They have also located the absolute-convective instability transition boundaries in the $(a, q)$-parameter plane by numerically applying the Briggs-Bers criterion to the inviscid dispersion relation. At $R e=667$, Delbende, Chomaz \& Huerre (1998) have also determined such transition curves from a nu- 
merical study of the linear impulse response. In both studies, a moderate amount of swirl is found to enlarge the region of absolute instability. Moreover, for jets, the azimuthal wavenumber of the convective-absolute transitional mode is found to be highly sensitive to the swirl level. By contrast, for wakes, the transitional mode is always $m= \pm 1$. According to the finite-Reynolds-number investigations of Delbende et al. (1998), viscous effects tend to reduce the domain of absolute instability both for jets and wakes.

The latter study of Delbende et al. (1998) is restricted to a single Reynolds number $(R e=667)$ and it is only capable of extracting, from the computed linear impulse response, the dominant mode that exhibits a convective-absolute transition. The objective of the present analysis is to document the effects of finite viscosity fully on spatial branches and on the extent of the domain of absolute instability by taking into account all amplified modes. Instead of working in physical space as in Delbende et al. (1998), we solve the linear dispersion relation directly by resorting to a Chebyshev spectral collocation method and subsequently tracking pinch points in the complex $k$-plane as dictated by the Briggs-Bers criterion.

The paper is organized as follows. The next section presents the governing eigenvalue problem of interest for this study. The spectral method used is described briefly in $\S 3$. Numerical results pertaining to spatial branches are discussed in $\S 4$. The absolute-convective instability transition curves are determined in the $(a, q)$-parameter plane for different Reynolds numbers in $\S 5$.

\section{Eigenvalue problem}

The basic flow is taken to be a vortex flow around the $x$-axis of typical core radius $R$ and angular swirl velocity $\Omega$. The axial flow is characterized by the centreline axial velocity $U_{c}$ and external axial velocity $U_{\infty}$. If we select as length scale the radius $R$ and as velocity scale the difference $\Delta U=U_{\infty}-U_{c}$, the Batchelor (1964) (a,q)-family of velocity profiles takes the form

$$
U(r)=a+\exp \left(-r^{2}\right), \quad V(r)=0, \quad W(r)=q\left[1-\exp \left(-r^{2}\right)\right] / r,
$$

where $(U, V, W)$ designate the velocity components in the usual non-dimensional coordinate system $(x, r, \theta)$. The linear stability analysis therefore involves three nondimensional control parameters: the axial co-flow parameter $a$, the swirl parameter $q$ and the Reynolds number $R e$, respectively defined by

$$
a=\frac{U_{\infty}}{\Delta U}, \quad q=\frac{\Omega R}{\Delta U}, \quad R e=\frac{\Delta U R}{v},
$$

where $v$ is the kinematic viscosity.

Typical azimuthal velocity profiles $W(r)$ are illustrated in figure $1(a)$ for two swirl values $q$. As shown in figures $1(b)-1(d)$, different settings for $a$ result in distinct axial flow configurations: co-flowing wakes (figure $1 b$ ) when $a<-1$, counter-flowing jets or wakes (figure $1 c$ ) when $-1<a<0$ and co-flowing jets (figure $1 d$ ) when $a>0$. By convention, the $(a, q)$-plane is divided into two regions: the 'jet' side for $a>-0.5$ and the 'wake' side for $a<-0.5$.

In classical fashion, the linear stability analysis consists in superimposing on the basic flow (2.1) velocity and pressure disturbances $\left(u^{\prime}, v^{\prime}, w^{\prime}, p^{\prime}\right)$ of the form

$$
\left(u^{\prime}, v^{\prime}, w^{\prime}, p^{\prime}\right)=\operatorname{Re}\left\{[H(r), \mathrm{i} F(r), G(r), P(r)] \mathrm{e}^{\mathrm{i}(k x+m \theta-\omega t)}\right\},
$$

where $H, F, G$ and $P$ are the eigenfunctions, $k=k_{r}+\mathrm{i} k_{i}$ is the complex axial 

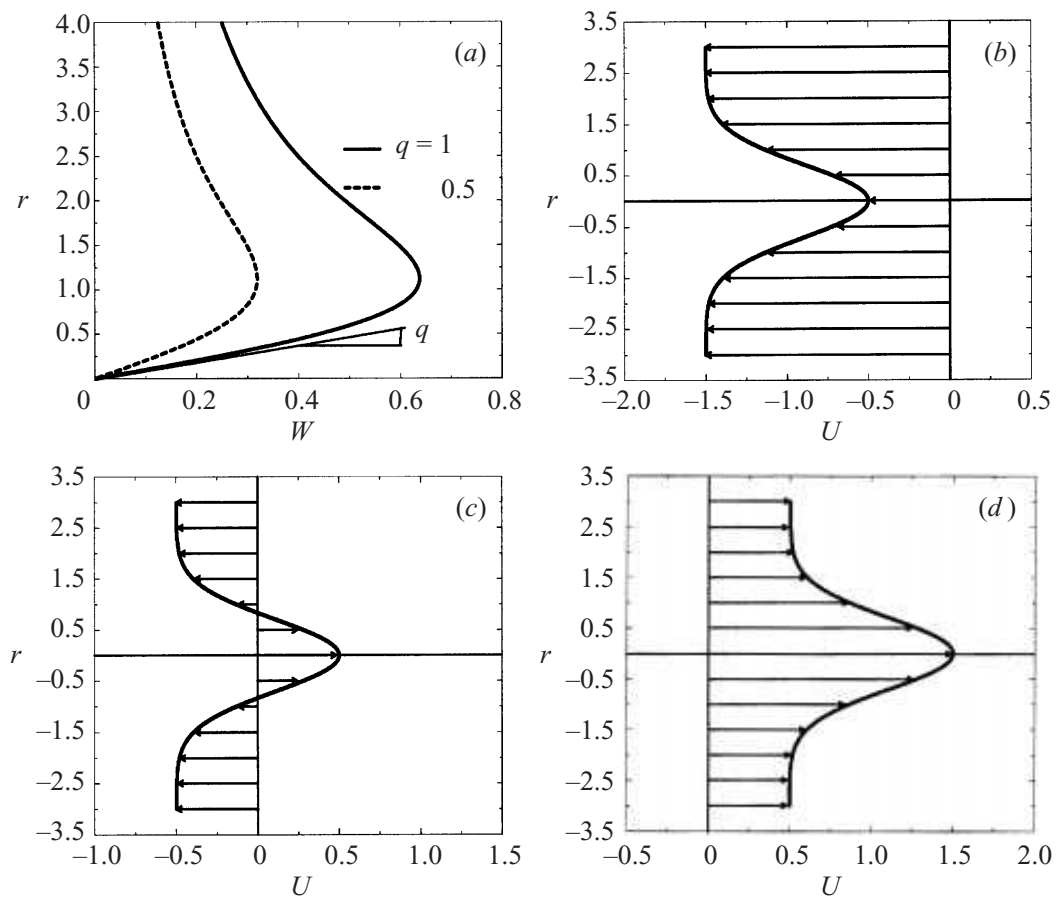

FIGURE 1. Batchelor vortex velocity profiles as a function of the swirl and co-flow parameters $q$ and a. (a) Azimuthal velocity profiles for $q=0.5$ and $q=1.0$. Axial velocity profiles for $(b)$ a co-flowing wake, $a<-1 ;(c)$ for a counter-flowing wake/jet $-1<a<0 ;(d)$ for a co-flowing jet, $a>0$.

wavenumber, $m$ is the integer azimuthal wavenumber and $\omega=\omega_{r}+\mathrm{i} \omega_{i}$ is the complex frequency. Upon substituting the modal decomposition (2.3) into the Navier-Stokes equations linearized about the basic flow (2.1), we obtain, as in Ash \& Khorrami (1995), the linear system of ordinary differential equations

$$
\begin{gathered}
F^{\prime}+\frac{F}{r}+\frac{m G}{r}+k H=0, \\
\frac{\mathrm{i} F^{\prime \prime}}{R e}+\frac{\mathrm{i} F^{\prime}}{r R e}-\left[\omega-\frac{m W}{r}-k U+\frac{\mathrm{i}\left(m^{2}+1\right)}{r^{2} R e}\right] F-2\left[\frac{\mathrm{i} m}{r^{2} R e}-\frac{W}{r}\right] G-P^{\prime}=\frac{\mathrm{i} k^{2} F}{R e}, \\
\frac{G^{\prime \prime}}{R e}+\frac{G^{\prime}}{r R e}+\left[\mathrm{i} \omega-\frac{\mathrm{i} m W}{r}-\mathrm{i} k U-\frac{m^{2}+1}{r^{2} R e}\right] G-\left[\mathrm{i} W^{\prime}+\frac{2 m}{r^{2} R e}+\frac{\mathrm{i} W}{r}\right] F-\frac{\mathrm{i} m P}{r}=\frac{k^{2} G}{R e}, \\
\frac{H^{\prime \prime}}{R e}+\frac{H^{\prime}}{r R e}+\left[\mathrm{i} \omega-\frac{\mathrm{i} m W}{r}-\mathrm{i} k U-\frac{m^{2}}{r^{2} R e}\right] H-\mathrm{i} U^{\prime} F-\mathrm{i} k P=\frac{k^{2} H}{R e}
\end{gathered}
$$

where primes denote $\mathrm{d} / \mathrm{d} r$. For the present unconfined flow case, this system is supplemented with the far-field conditions

$$
F(\infty)=G(\infty)=H(\infty)=P(\infty)=0,
$$


while the requirement that all perturbations be bounded and single-valued on the centreline imposes (see Batchelor \& Gill 1962; Mayer \& Powell 1992)

$$
\begin{gathered}
F(0)=G(0)=H(0)=P(0)=0 \quad \text { if } \quad m^{2}>1, \\
H(0)=P(0)=0, \quad F^{\prime}(0)=G^{\prime}(0)=0 \quad \text { if } \quad m^{2}=1, \\
F(0)=G(0)=0, \quad H(0) \text { and } P(0) \quad \text { finite if } m^{2}=0 .
\end{gathered}
$$

For given control parameters $(a, q, R e)$ and azimuthal wavenumber $m$ the system (2.4)-(2.11) constitutes an eigenvalue problem whereby a non-zero solution $(F, G, H, P)$ exists if and only if the complex pair $(k, \omega)$ satisfies the dispersion relation $\mathscr{D}(k, \omega ; m, a, q, R e)=0$. The present work focuses on the following spatial problem: for a given complex frequency $\omega$, solutions of the dispersion relation are sought in terms of spatial branches $k(\omega ; m, a, q, R e)$ and their associated eigenfunctions. System (2.4)-(2.11) is readily seen to be invariant under the transformations $(a, m, q) \longrightarrow(a,-m,-q),(H, F, G, P) \longrightarrow(H, F,-G, P)$. Thus, without loss of generality, we may restrict the investigation to positive values of the swirl parameter $q$ and allow both positive and negative values of the azimuthal wavenumber $m$. This usual restriction will be adopted in the presentation of the spatial instability characteristics. However, in the discussion of the absolute/convective instability transition curves we have found it advantageous to allow for negative swirl values as well, in order to track the same azimuthal mode.

It is well established (see, for instance, Huerre \& Rossi 1998) that the strictly spatial instability analysis ( $\omega$ given real, $k$ unknown complex) effectively describes the large-time spatial response to a periodic excitation of frequency $\omega$ applied at $x=0$, only if the basic flow is convectively unstable. In this context, the response typically consists of spatial branches $k^{+}(\omega)$ and $k^{-}(\omega)$, respectively, located on the right- $(x>0)$ and left- $(x<0)$ hand sides of the periodic source. The distinction between $k^{+}$and $k^{-}$branches is no longer tenable in an absolutely unstable basic flow when the spatial response to a time-periodic excitation becomes ill-posed. Provided that convective instability prevails, the $k^{+}$and $k^{-}$branches are spatially amplified away from the source at $x=0$ if $-k_{i}^{+}>0$ and $k_{i}^{-}>0$, respectively. On the 'jet' side $(a>-0.5)$, the flow is predominantly to the right $(x>0)$ and the modes $k^{-}$and $k^{+}$are the 'upstream' and 'downstream' branches, respectively. On the 'wake' side, $(a<-0.5)$, the flow is predominantly to the left-hand side $(x<0)$ and the $k^{-}$and $k^{+}$branches switch roles, $k^{-}$becoming the downstream branch and $k^{+}$the upstream branch.

The appearance at a specific parameter setting $(m, a, q, R e)$ of a pinch point between $k^{+}$and $k^{-}$branches for a real absolute frequency $\omega_{0}$ indicates a transition from convective to absolute instability (see for instance Huerre \& Rossi 1998). The determination of such a pinch point which satisfies $\omega_{0, i}=0$ is sufficient to determine the absolute-convective instability transition curves in the $(a, q)$-plane for given $m$ and $R e$. The existence of a similar pinch point for a complex absolute frequency $\omega_{0}=\omega_{0, r}+\mathrm{i} \omega_{0, i}$ allows a rigorous distinction between absolute $\left(\omega_{0, i}>0\right)$ and convective $\left(\omega_{0, i}<0\right)$ instability. According to the Briggs-Bers criterion, the complex pair $\left(k_{0}, \omega_{0}\right)$ is given by the zero-group velocity condition $\partial \omega / \partial k\left(k_{0}\right)=0, \omega_{0}=\omega\left(k_{0}\right)$. Furthermore, the only relevant complex pair $\left(k_{0}, \omega_{0}\right)$ is the one that first involves the pinching of two $k^{+}$and $k^{-}$branches that emerge from distinct upper and lower half- $k$-planes as $\omega_{i}$ is decreased from large positive values. 


\section{Numerical procedure}

According to Khorrami, Malik \& Ash (1989), the eigenvalue problem (2.4)-(2.11) is accurately and efficiently solved by resorting to a Chebyshev collocation method, as described in Gottlieb \& Orszag (1977). This approach has been successively applied by Khorrami (1991) and Mayer \& Powell (1992) to the temporal problem ( $k$ given real, $\omega$ unknown complex). In the present context, the collocation method is implemented for the spatial problem ( $\omega$ given complex, $k$ unknown complex). See Khorrami et al. (1989) for a detailed presentation of the numerical procedure.

In order to reduce system (2.4)-(2.11) to a linear matrix eigenvalue problem, a generalized eigenvector $X=(F, G, H, k F, k G, k H, P)$ is introduced and the far-field boundary conditions (2.8) are enforced at a large but finite radius $r_{\max } \gg 1$. The Chebyshev collocation method is known to concentrate most collocation points in the vicinity of $r=0$ and $r_{\max }$. In order to circumvent this difficulty, the two-parameter tranformation of Malik, Zang \& Hussaini (1985)

$$
\frac{r}{r_{c}}=[1+\eta] /\left[1-\eta+\frac{2 r_{c}}{r_{\max }}\right]
$$

is introduced, which maps the Chebyshev interval $-1 \leqslant \eta \leqslant+1$ onto the physical domain $0 \leqslant r \leqslant r_{\max }$. Note that the parameter $r_{c}$ in (3.1) allows half the points in the central shear region $0 \leqslant r \leqslant r_{c}$ to be distributed. Upon rewriting system (2.4)-(2.11) in terms of the $\eta$-variable and discretizing it, we arrive at the generalized eigenvalue problem

$$
\boldsymbol{A} \boldsymbol{X}=k \boldsymbol{B} \boldsymbol{X},
$$

for a discretized eigenvector $\boldsymbol{X}=\boldsymbol{X}(\eta)$. If $N$ denotes the number of collocation points, matrices $\boldsymbol{A}$ and $B$ are $7 N \times 7 N$. Furthermore, since $k^{2}$ only appears in equations (2.5)-(2.7), the matrix $\boldsymbol{B}$ is singular. The discretized problem is subsequently solved by using the SCILIB routines CGEGS and CGEVS. The Schurr decomposition algorithm implemented in the routines CGEGS and CGEVS yields the spectrum $\mathscr{S}_{N}$ of eigenvalues $k$ for the discretized problem (3.2) and the generalized eigenvectors $X$. Owing to the discretization, most elements of $\mathscr{S}_{N}$ are found to be spurious eigenvalues. As in the temporal investigation of Mayer \& Powell (1992), the location of the spurious modes in the complex $k$-plane is highly sensitive to the discretization parameter $N$, in contrast to the few physical eigenvalues of the problem. Physical modes are readily extracted by comparing spectra $\mathscr{S}_{N}$ and $\mathscr{S}_{N}^{\prime}$ for $N^{\prime}>N$. As discussed in Huerre \& Monkewitz (1985), attention is restricted to the right-hand half-plane $\operatorname{Re}(k)>0$. Hence, $k^{\prime}$ of $\mathscr{S}_{N}^{\prime}$ is selected if $\operatorname{Re}\left(k^{\prime}\right)>0$ and $\operatorname{Min}\left|k^{\prime}-k\right| \leqslant \epsilon$ for $k \in \mathscr{S}_{N}$. All computations have been performed on a CRAY C90 computer using 64-bit precision real and 128-bit complex arithmetic with $\epsilon=10^{-3}$.

The computational sensitivity of two primary spatial modes to changes in the parameters $N$ and $r_{\max }$ is illustrated in tables 1 and 2, respectively. The selected settings $N=80$ and $r_{\max }=100$ are seen to provide the eigenvalues with a five-digit accuracy, which is deemed to be sufficient for the present purposes. The validity of the present Chebyshev spectral collocation approach has been checked by comparing our spatial viscous mode results with those obtained by Khorrami (1991) within a temporal framework. For instance, at $R e=10.000$, Khorrami (1991) has obtained a viscous bending $(m=+1)$ mode of complex frequency $\omega=0.32345326-0.00009192 \mathrm{i}$ at the real axial wavenumber $k=0.3$. With the present spatial method, we detect a viscous bending mode of complex wavenumber $k=0.29999826+0.00005638 \mathrm{i}$ at the same complex frequency $\omega$. 


\begin{tabular}{rccc}
$N$ & Mode 1: $\omega=0.1$ & & Mode 2: $\omega=-0.15$ \\
\cline { 2 - 2 } 70 & $0.669367151-0.181999974 \mathrm{i}$ & & $0.367202058-0.220458484 \mathrm{i}$ \\
70 & $0.669365472-0.181997057 \mathrm{i}$ & & $0.367202508-0.220457875 \mathrm{i}$ \\
80 & $0.669363836-0.181994107 \mathrm{i}$ & & $0.367202981-0.220457371 \mathrm{i}$ \\
100 & $0.669360884-0.181988583 \mathrm{i}$ & & $0.367203916-0.220456594 \mathrm{i}$
\end{tabular}

TABLE 1. Convergence behaviour of complex wavenumber $k=k_{r}+\mathrm{i} k_{i}$ for the two 'primary' bending modes $m=-1$ as a function of the number $N$ of Chebyshev polynomials. $a=0, q=0.7, R e=667$, $r_{c}=3, r_{\max }=100$.

\begin{tabular}{rccc}
$r_{\max }$ & Mode 1: $\omega=0.1$ & & Mode $2: \omega=-0.15$ \\
\cline { 2 - 2 } 100 & $0.669361381-0.181989583 \mathrm{i}$ & & $0.367203729-0.220456678 \mathrm{i}$ \\
120 & $0.669363836-0.181994107 \mathrm{i}$ & & $0.367202981-0.220457371 \mathrm{i}$ \\
$0.669365149-0.181996458 \mathrm{i}$ & & $0.367202602-0.220457782 \mathrm{i}$
\end{tabular}

TABLE 2. Convergence behaviour of complex wavenumber $k=k_{r}+\mathrm{i} k_{i}$ for the two 'primary' bending modes $m=-1$ as a function of the position $r_{\max }$ where far-field conditions are enforced. $a=0$, $q=0.7, \operatorname{Re}=667, r_{c}=3, N=80$.

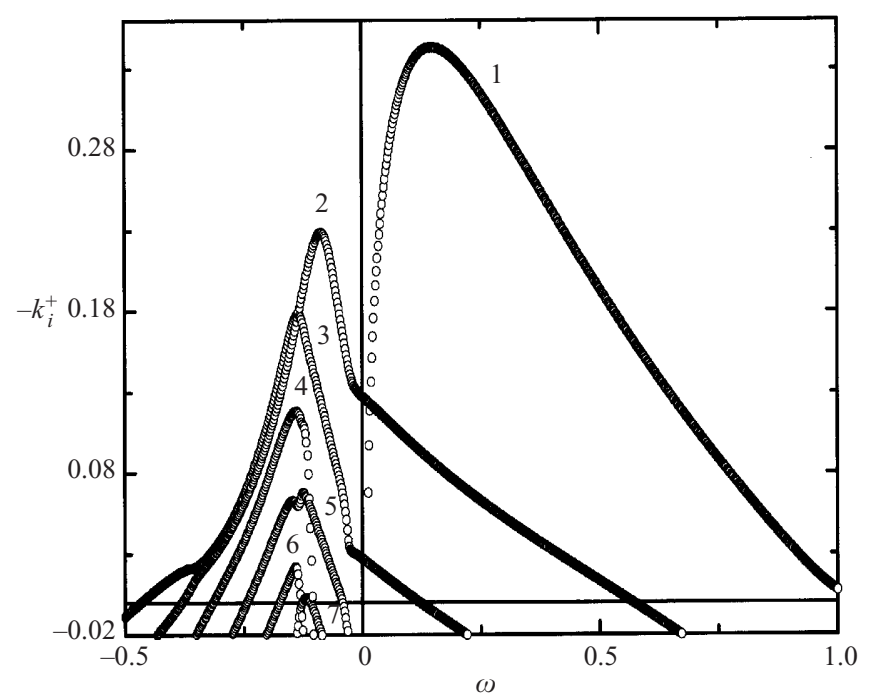

FIGURE 2. Spatial growth rate $-k_{i}^{+}$of bending modes $m=-1$ versus real frequency $\omega$ for $a=0, q=0.6$ and $R e=2000$. The mode number is indicated next to each curve. The swirl is zero on the horizontal solid line.

\section{Spatial branches in a convectively unstable domain}

In this section, only strictly spatial branches ( $\omega$ real, $k$ complex $)$ are considered for zero-external flow jets $(a=0)$, in situations where they are convectively unstable, as ascertained in $\S 5$. Attention is restricted to the downstream branch $k^{+}$, which is the only one to experience amplification away from the source.

The variations of the spatial growth rate $-k_{i}^{+}(\omega)$ with real frequency $\omega$ are represented in figure 2 for the bending mode $m=-1$ of a swirling jet at $q=0.6$ and $R e=2000$. Seven $k^{+}$-modes are seen to be amplified, with mode 2 crossing modes 1 and 3 at specific values of $\omega$. As a result, the 'primary' mode, i.e. the most amplified 


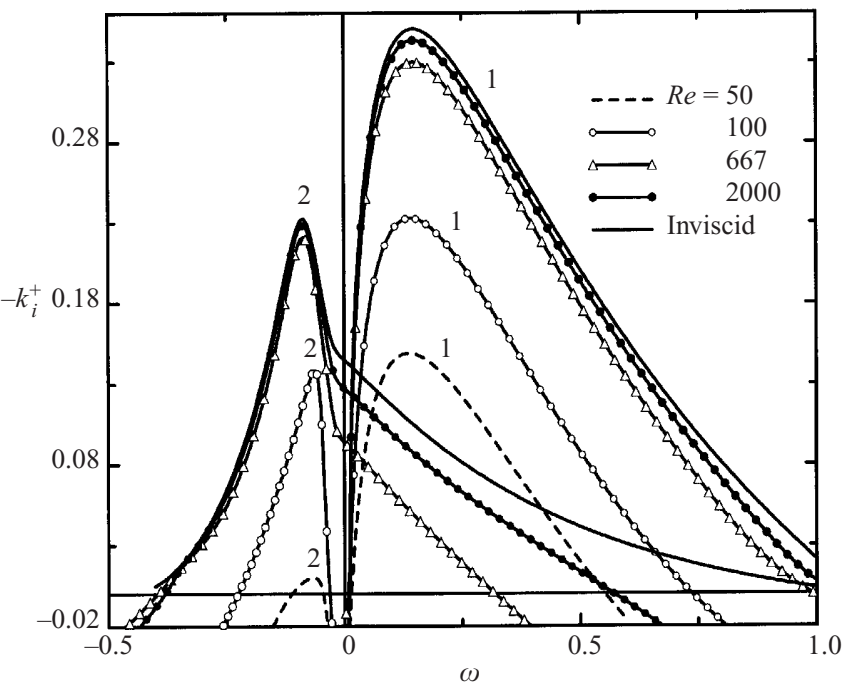

FIGURE 3. Spatial growth rate $-k_{i}^{+}$of 'primary' bending modes 1 and 2 for different Reynolds numbers and $a=0, q=0.6, m=-1$. The mode number is indicated next to each curve. The swirl is zero on the horizontal solid line.

\begin{tabular}{cccccccc}
\hline Mode & 1 & 2 & 3 & 4 & 5 & 6 & 7 \\
$\operatorname{Re}_{e}$ & 20.9 & 47.6 & 167.4 & 428 & 861.2 & 1521 & 1931
\end{tabular}

TABLE 3. 'Emergence' Reynolds number $R e_{e}$ pertaining to different spatially amplified and inviscid $k^{+}$branches of azimuthal wavenumber $m=-1$ for $a=0, q=0.6$.

one, is not always the same across the entire frequency range; as $\omega$ increases, it switches from mode 3 to mode 2 to mode 1 . Note that modes 1 and 2 are those previously considered in tables 1 and 2 for $q=0.7$ and $R e=667$.

The effect of decreasing viscosity is illustrated in figure 3. At the low-Reynoldsnumber setting $R e=50$, only bending modes 1 and 2 are spatially amplified. As $R e$ increases above 50, additional amplified modes arise beyond specific 'emergence' Reynolds numbers $R e_{e}$. Such 'emergence' Reynolds numbers are given in table 3 for different modes $1,2,3, \ldots$ In the range $50<R e<2000$, the mode hierarchy remains the same as in figure 2, but modes 2 and 3 not displayed in figure 3 cross as $R e$ exceeds 1580 . The unstable frequency range of mode 2 is seen to expand very rapidly as $R e$ increases whereas it is only slightly affected for mode 1. For the moderate swirl value $q=0.6$, each curve gradually approaches the inviscid limit determined by Olendraru et al. $(1996,1999)$, as the Reynolds number is increased. The same behaviour holds for all other modes 3 to 7 . It is therefore legitimate to call such modes 'inviscid' since they reach their maximum amplification for vanishing viscosity. Finally, note that additional 'inviscid' modes may become destabilized as $R e$ exceeds 2000.

It should be emphasized that numerical resolution difficulties may arise in the determination of 'inviscid' spatial branches at large Reynolds numbers whenever critical layers are encountered. According to Olendraru et al. (1999), each inviscid mode of the Batchelor vortex may exhibit zero, one or two non-zero complex critical 


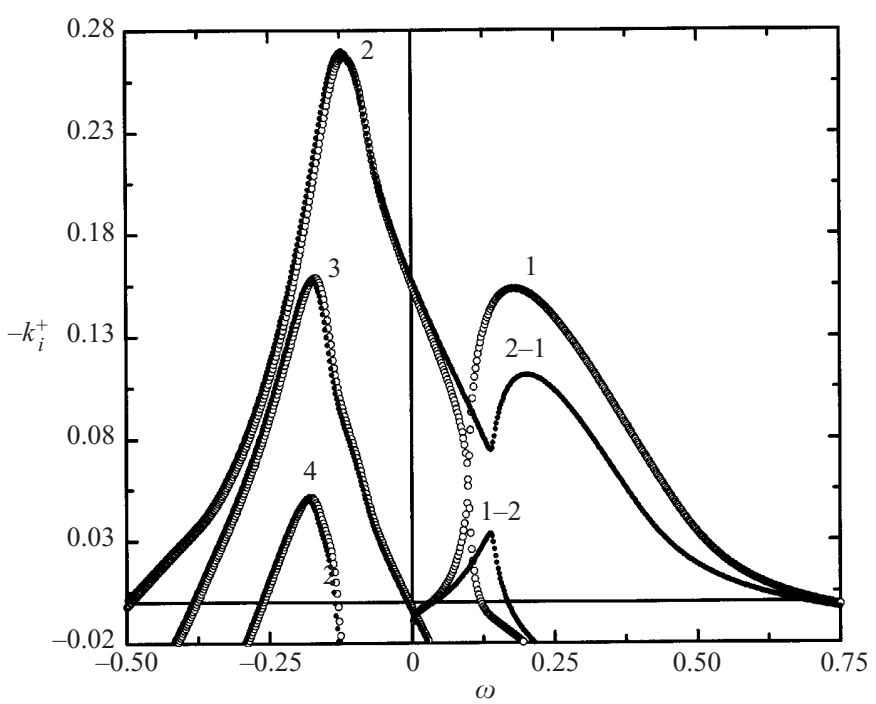

FIGURE 4. Spatial growth rate $-k_{i}^{+}$of bending modes $m=-1$ versus the frequency $\omega$ for $a=0$ and $R e=667$. Mode switching takes place as $q$ changes from $O, q=0.725$ to $\bullet, q=0.74$. The mode number is indicated next to each curve. The swirl is zero on the horizontal solid line.

points $z_{c}$ in the complex half-plane $\operatorname{Re}\left(z_{c}\right) \geqslant 0$ defined by

$$
k U\left(z_{c}\right)-\omega+m W\left(z_{c}\right) / z_{c}=0 .
$$

As a result, a so-called 'viscous sector' associated with the critical point may contain part of the physical real axis $0<r<r_{\max }$. In such intervals of $r$, the eigenfunctions are viscous-like and therefore display large oscillations. This feature is accentuated as $R e$ increases and may be inadequately resolved unless additional collocation points are introduced. In order to circumvent this resolution issue, Mayer \& Powell (1992) chose, in their investigation of the viscous temporal problem, to deform the integration contour around the critical point(s) in the complex $r$-plane. Olendraru et al. (1999) resorted to the same procedure in the inviscid spatial analysis. In the present instance, we have chosen to stay on the real $r$-axis.

The effect of increasing swirl is depicted in figure 4 for $R e=667$. As $q$ increases from 0.6 , the formerly denoted mode 2 becomes more amplified than mode 1 (compare figures 2 and 4). Moreover, a switching phenomenon takes place between the 'primary' modes 1 and 2 as the swirl increases from $q=0.725$ to $q=0.74$. As a result, two hybrid modes 2-1 and 1-2 emerge to replace the original modes 1 and 2. Observe that the unstable frequency range of the hybrid mode $2-1$ is large, which is not the case for the hybrid mode 1-2. Mode switching has also been encountered in the temporal case, as reported by Khorrami (1992) for the Batchelor vortex and Cotton \& Salwen (1981) for Hagen-Poiseuille flow.

For a given parameter setting ( $m, a, q, R e)$, each spatial mode may be characterized by a maximum spatial growth rate $\left(-k_{i}^{+}\right)_{\max }$ over all real frequencies. The variations of $\left(-k_{i}^{+}\right)_{\max }$ with positive swirl $q$ are illustrated in figure 5 for the unstable modes $1,2,1-2,2-1,3,4$ at $m= \pm 1$ and $R e=667$. According to table 3, modes 5, 6 and 7 are stable at $R e=667$ for $q=0.7$. It has been checked that these modes remain stable in the entire swirl range. Note the occurrence of an inviscid $m=+1$ mode which is simply obtained from its $m=-1$ counterpart by reflection with respect to 


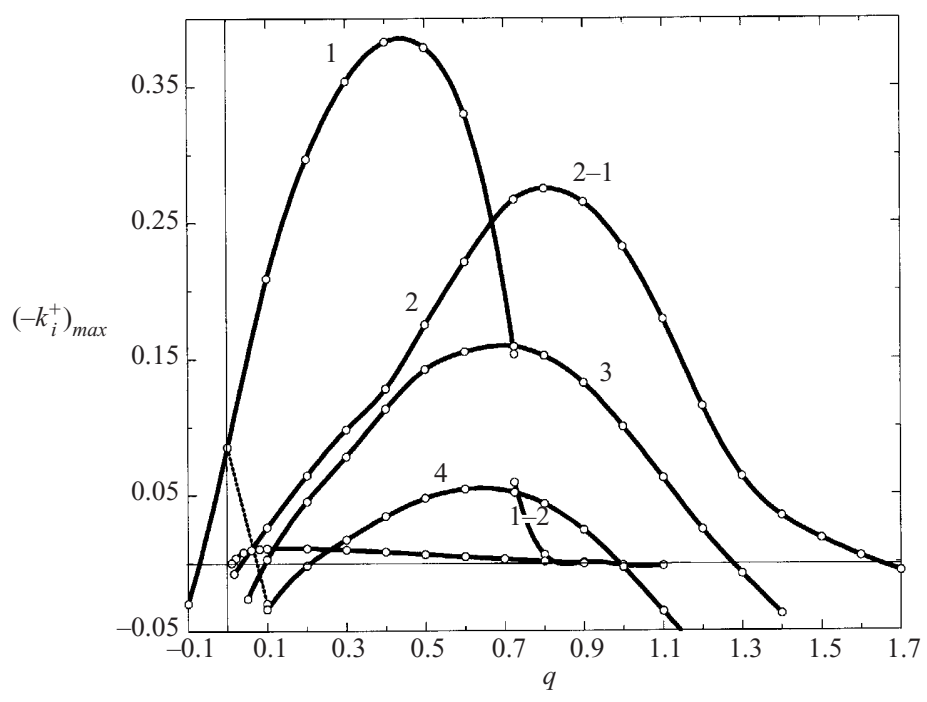

FIGURE 5. Maximum spatial growth rate $\left(-k_{i}^{+}\right)_{\max }$ of bending modes $m=-1$ versus swirl parameter $q$ for $a=0$ and $R e=667$. The inviscid mode number is indicated next to each solid line. The dashed line pertains to the viscous mode appearing at negative swirl values. The swirl is zero on the horizontal solid line. The dotted line pertaining to the $m=+1$ inviscid mode has been included for completeness. It is obtained from its $m=-1$ counterpart by reflection with respect to the vertical axis.

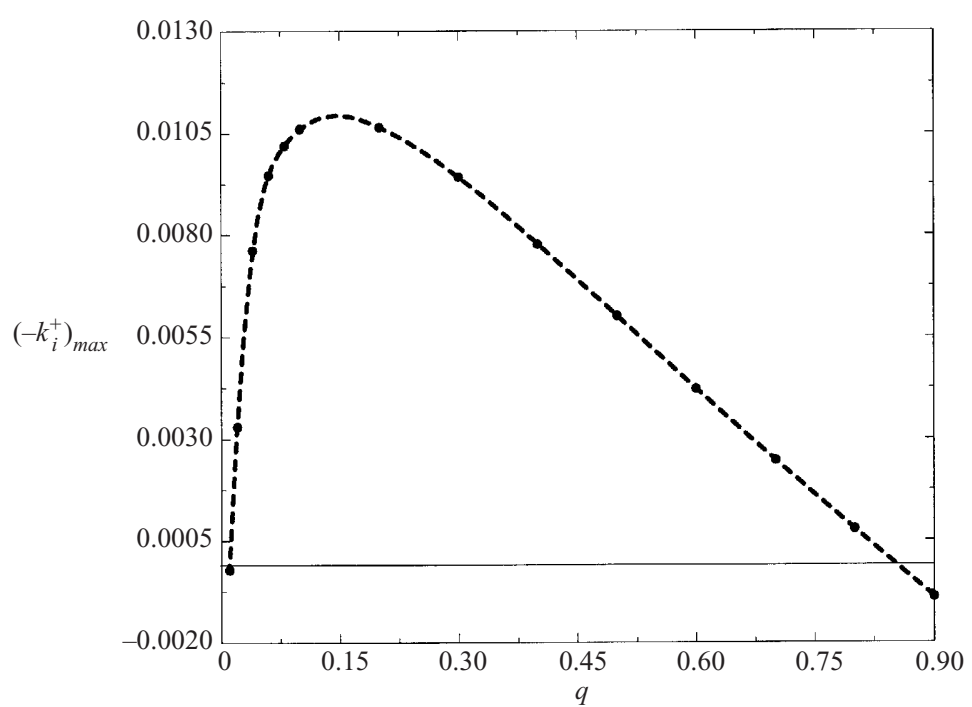

FIGURE 6. Maximum spatial growth rate $\left(-k_{i}^{+}\right)_{\max }$ of viscous bending mode $m=+1$ versus swirl parameter $q$ for $a=0$ and $R e=667$. Zoom of viscous mode in figure 5. Note the weak amplification. The swirl is zero on the horizontal solid line.

the vertical axis. Furthermore, a new weakly amplified branch is seen to appear also at $m=+1$.

As displayed in figure 6, its maximum growth rate is an order of magnitude below that of inviscid modes. The spatial growth rate of this mode is displayed in figure 7 as a function of frequency for various Reynolds numbers. It is readily observed 


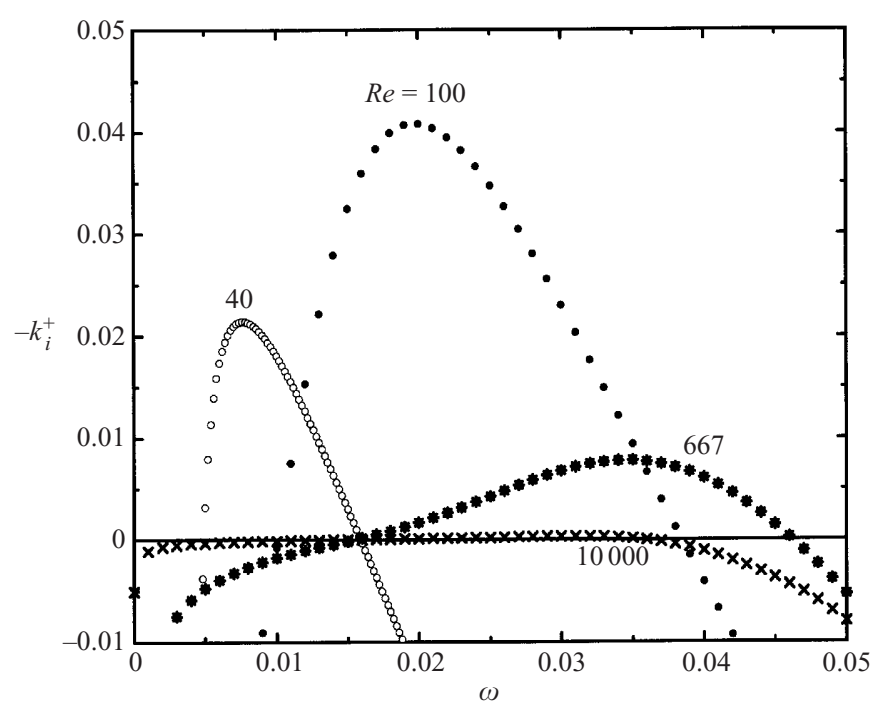

FIGURE 7. Spatial growth rate $-k_{i}^{+}$of viscous bending mode $m=+1$ versus real frequency $\omega$ for different Reynolds numbers $R e$ at $a=0, q=0.4$. O, $R e=40 ; \bullet, R e=100 ; *, R e=667 ; \times$, $R e=10000$.

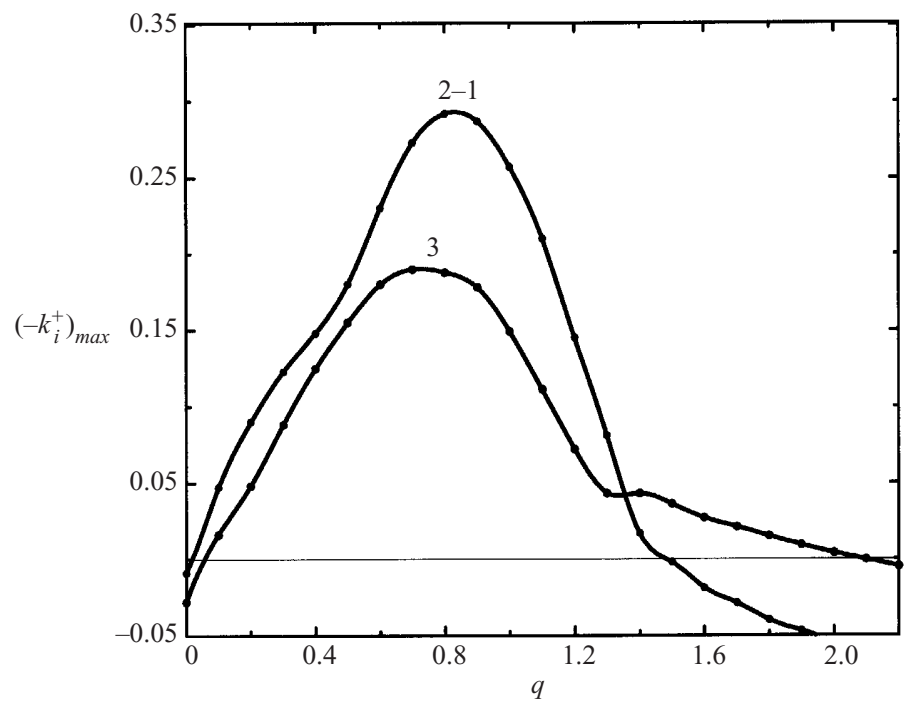

FIgURE 8. Maximum spatial growth rate $\left(-k_{i}^{+}\right)_{\max }$ of bending modes $m=-1$ (mode 3 and hybrid mode 2-1) versus swirl parameter $q$ for $a=0$ and $R e=2000$. The inviscid mode number is indicated next to each solid curve. The swirl is zero on the horizontal solid line.

that amplification levels uniformly decrease to zero as the Reynolds number roughly exceeds 10000 . Conversely, growth rates also decrease as viscous effects become too large, e.g. at $R e=40$. It is therefore concluded that the weakly amplified $m=+1$ mode at moderate Reynolds numbers is indeed of viscous nature. It has been verified that it effectively connects at the neutral frequency to the temporal viscous mode first identified by Khorrami (1991) and Mayer \& Powell (1992). As observed in figure 5, all of the inviscid modes are damped as $q$ exceeds the critical value $q_{c} \sim 1.6$, for 


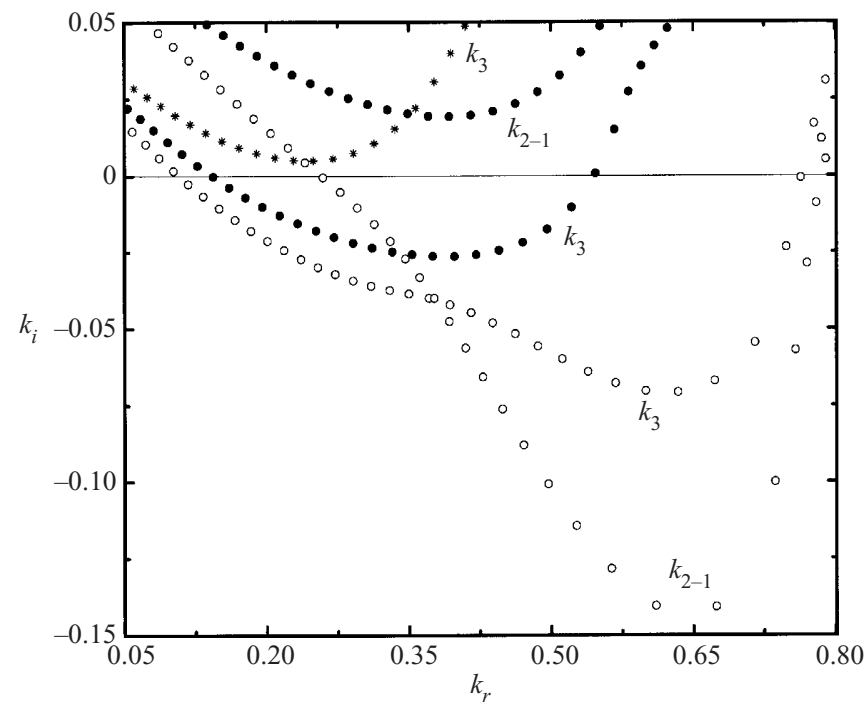

FIGURE 9. Loci of spatial bending modes $m=-1$ (mode 3 and hybrid mode 2-1) in complex $k$-plane as a function of real frequency $\omega$ for $a=0$ and $\operatorname{Re}=2000$. $\bullet, q=1.2$; $0, q=1.6$; *, $q=2.2$.
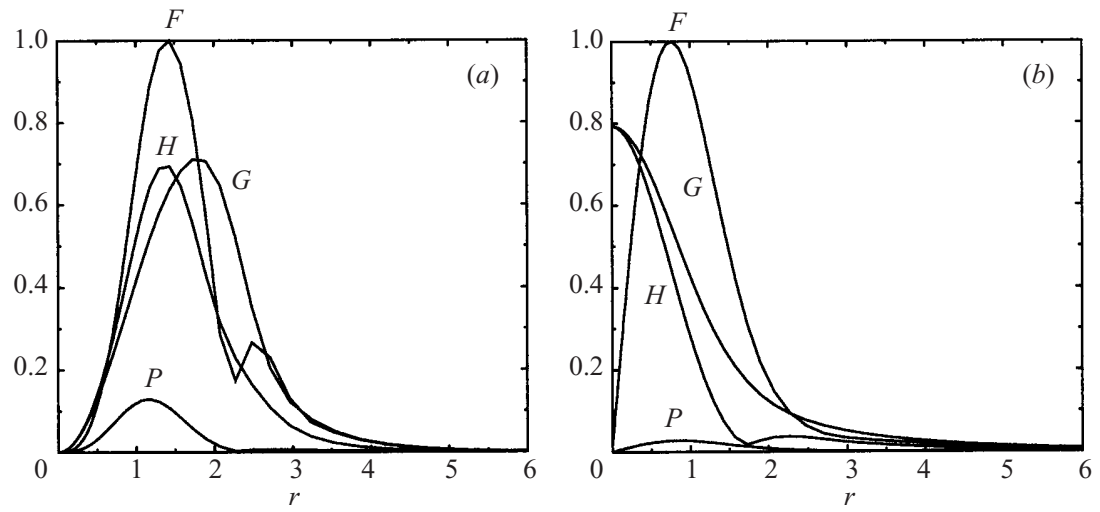

FIGURE 10. Spatial eigenfunction amplitudes $|F(r)|,|G(r)|,|H(r)|$ and $|P(r)|$ for co-flowing jets at $R e=667$. Parameter settings in $(a)$ and $(b)$ are approximately indicated by the symbol $*$ in figure 21. (a) Case $m=-3, a=0.002, q=0.4, \omega=-0.22$ and $k_{1}^{+}=(1.443,-1.481)$. $(b)$ Viscous bending mode $m=+1, a=0, q=0.4, \omega=0.035$ and $k^{+}=(0.371,-0.078)$.

$R e=667$. As the Reynolds number increases, the critical swirl value $q_{c}$ also increases. For instance, according to figure 8 , at the larger setting $R e=2000$, mode 3 still remains weakly amplified at $q_{c} \sim 2.1$ while mode $2-1$ stays damped. The peculiar behaviour of modes $2-1$ and 3 at $R e=2000$ is further illustrated in the complex $k$-plane of figure 9 for three swirl values $q=1.2,1.6$ and $2.2>q_{c}$. As $q$ increases from 1.2 to 1.6 , the hybrid mode $2-1$ is rapidly stabilized at large $k_{r}$ while only minimal variation with $q$ take place at small $k_{r}$.

Typical radial distributions of spatial eigenfunction amplitudes are displayed in figures 10-12 for increasing Reynolds numbers. The normalization condition is such that the maximum amplitude over all radial stations and all physical components 

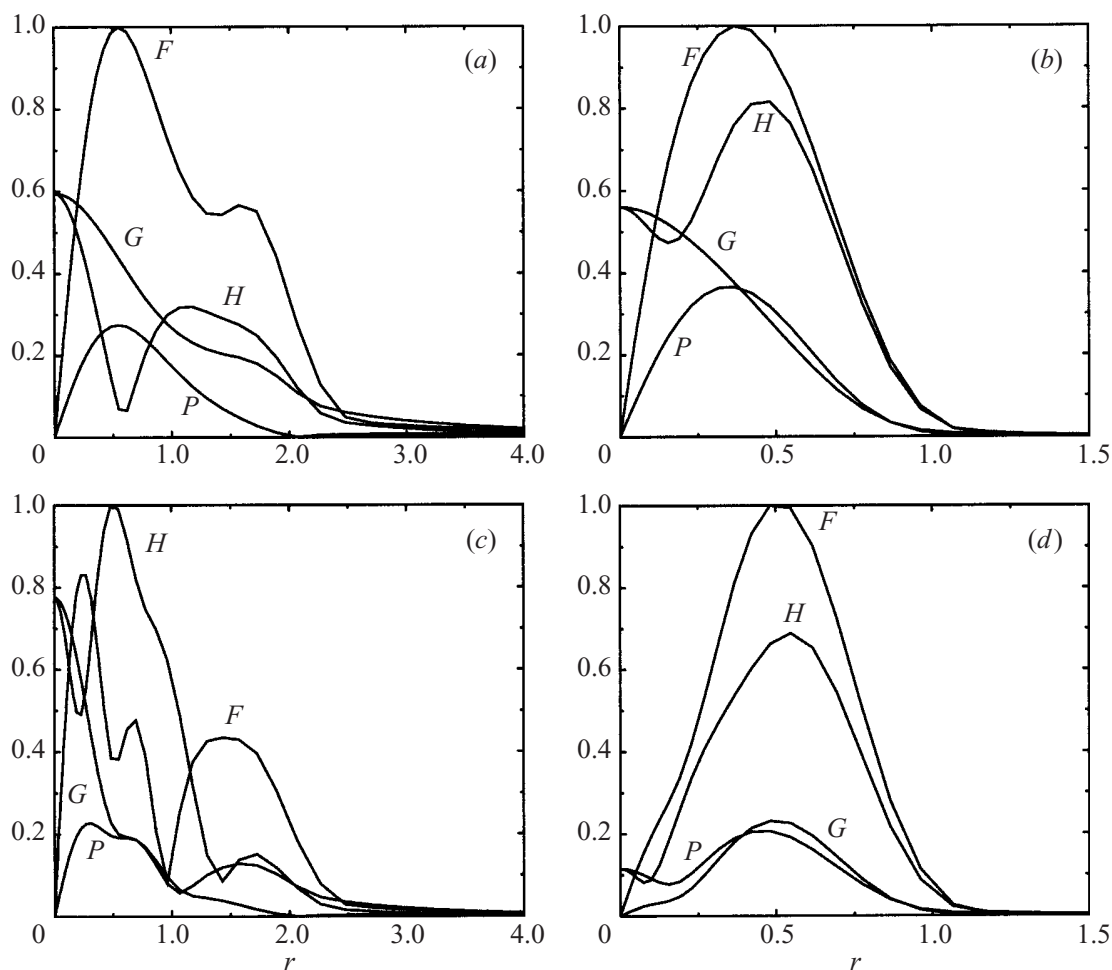

FIGURE 11. Spatial eigenfunction amplitudes $|F(r)|,|G(r)|,|H(r)|$ and $|P(r)|$ of bending mode $m=-1$ for a zero co-flow jet at $R e=2000$. ( $a) q=0.8, \omega=-0.2$ and $k_{2-1}^{+}=(0.387,-0.222)$. (b) $q=1.6$, $\omega=-1.05$ and $k_{2-1}^{+}=(0.361,-0.02)$. (c) $q=0.8, \omega=-0.2$ and $k_{3}^{+}=(0.665,-0.133)$. (d) $q=1.6$, $\omega=-1.05$ and $k_{3}^{+}=(0.352,-0.025)$.

$F, G, H$ and $P$ is unity. An $m=-3$ inviscid mode and an $m=+1$ viscous mode are represented in figures $10(a)$ and $10(b)$, respectively, for a co-flowing jet at $R e=667$. By anticipation, parameter settings for $a$ and $q$ have been chosen to lie on the convectively unstable side of the absolute-convective transition boundary at $R e=667$ as indicated by the star symbol in figure 21 . The perturbations are seen to be confined within a cylinder of approximate radius $r=5$. The $m=-1$ eigenfunctions pertaining to the 'inviscid' modes $k_{2-1}^{+}$and $k_{3}^{+}$of figure 9 are plotted in figure 11 at two distinct swirl parameter settings $q=0.8$ in figures $11(a)$ and $11(c)$ and $q=1.6$ in figures $11(b)$ and $11(d)$ for a zero co-flow jet $(a=0)$ at $R e=2000$. As $q$ increases, the radial extent of each mode shrinks while their spatial growth rate decreases. In other words, for large $q$, such disturbances tend to become centre-modes confined in the vicinity of the vortex axis, as in the temporal theory of Stewartson \& Brown (1985). Finally, various examples of eigenfunctions are depicted in figure 12 for the large Reynolds number $R e=10000$. By anticipation, parameter settings for $a$ and $q$ in figure 12 have been selected to lie on the convectively unstable side of the absolute-convective transition boundary at $R e=10000$, as indicated by the labelled star symbols in figure 24. In figure $12(a)$ and $12(b)$, the $m=-1$ mode for two counterflow jets at moderate swirl levels is displayed. In figures $12(c)$ and $12(d)$, the $m=-1$ mode (figure $12 c$ ) and the $m=-2$ mode (figure 12d) are represented for two co-flow wakes at large swirl levels. Here again, modes tend to become centred as $q$ increases. 

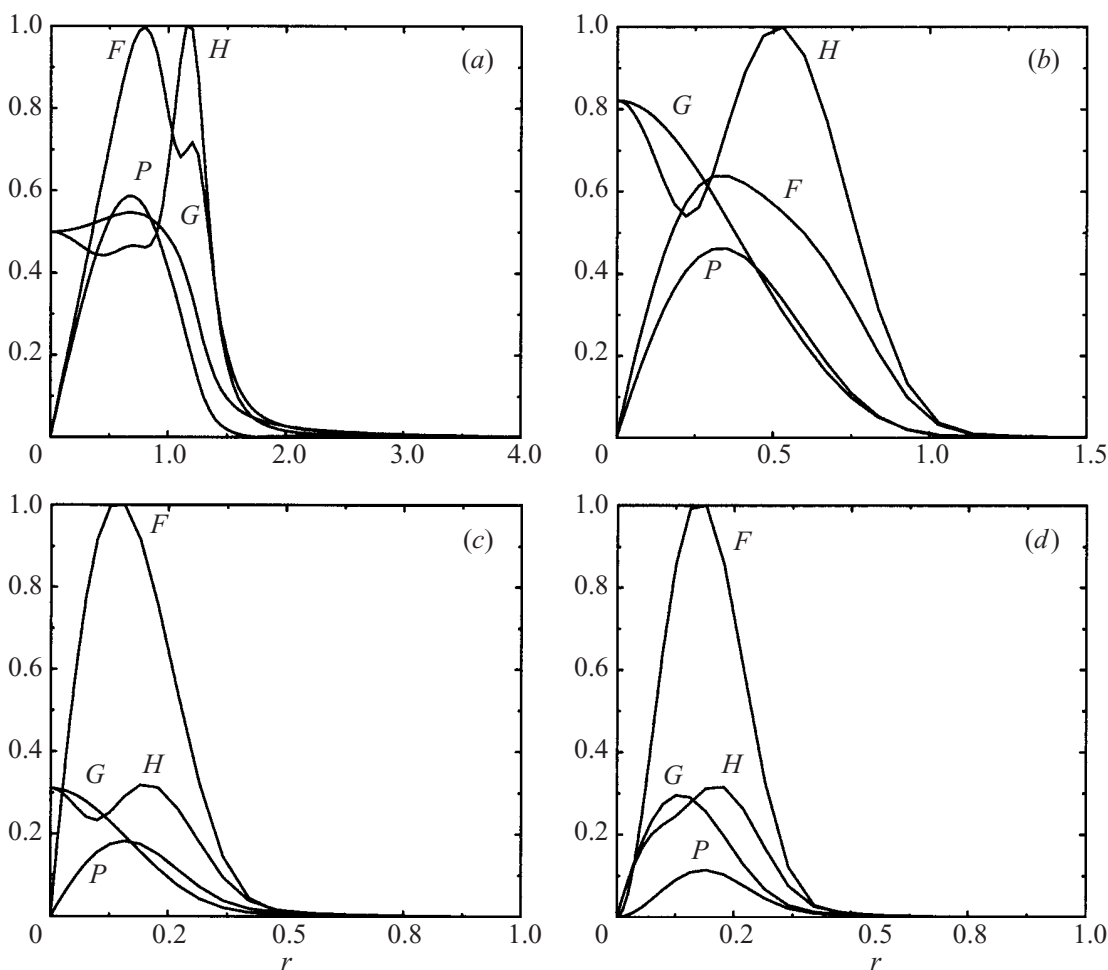

FIGURE 12. Spatial eigenfunction amplitudes $|F(r)|,|G(r)|,|H(r)|$ and $|P(r)|$ at $R e=10000$. (a) $m=-1$, counter-flowing jet $a=-0.05, q=1.2, \omega=-0.49$ and $k_{2-1}^{+}=(0.935,-0.177)$. (b) $m=-1$, counter-flowing jet $a=-0.2, q=1.41, \omega=-0.84$ and $k_{2-1}^{+}=(0.687,-0.0283)$. (c) $m=-1$, co-flowing wake $a=-1.17, q=3.1, \omega=-3.05$ and $k^{-}=(0.163,0.0098)$. (d) $m=-2$, co-flowing wake $a=-1.09, q=2.5, \omega=-4.92$ and $k^{-}=(0.488,0.055)$. Parameter settings in $(a)-(d)$ are indicated by labelled star symbols in figure 24 .

\section{Transition from convective to absolute instability}

In the previous section, spatial branches were determined for a zero external flow jet $(a=0)$ at parameter settings giving rise to convective instability only. When $a$ is varied, a transition to absolute instability may occur as discussed below. At given values of the parameters $m, q$ and $R e$, the external flow parameter $a$ is varied so as to detect the saddle point $k_{0}$ in the complex $k$-plane which involves pinching of two distinct spatial branches $k^{+}$and $k^{-}$emerging from the upper and lower half- $k$-plane, respectively. In order to monitor the deformation of spatial branches, it is sufficient to extend the previous determination of spatial branches to complex values of $\omega$.

A typical saddle point behaviour is illustrated in figure 13 for the azimuthal wavenumber $m=-3$ at the absolute-convective instability transition point $a=$ $0.0021, q=0.4$ and $R e=667$. The level contours $\omega_{i}=0$ of figure 14 demonstrate the existence of a saddle point $k_{0}$ between two spatial branches $k_{1}^{+}$and $k^{-}$. Other non-pinching less-amplified branches $k_{2}^{+}, k_{3}^{+}$and $k_{4}^{+}$are also displayed. It is indeed verified in figure 14 that the spatial branches $k_{1}^{+}$and $k^{-}$represented by different level contours $\omega_{i}=$ const $\geqslant 0$ do receed into their respective upper and lower half- $k$-plane as $\omega_{i}$ increases. The saddle point $k_{0}$ therefore signals a genuine transition to absolute instability.

The same procedure has been implemented to generate the entire absolute- 


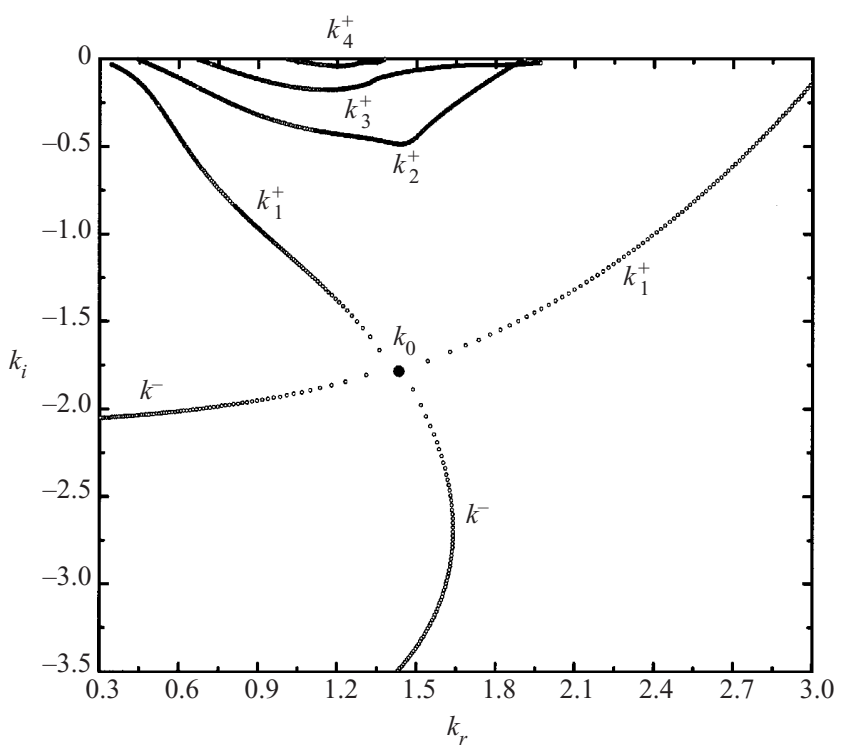

FIGURE 13. Saddle point $k_{0}$ in the complex $k$-plane for mode $m=-3$ at $a=0.0021, q=0.4$ and $R e=667$. In addition to the pinching branches $k_{1}^{+}$and $k^{-}$, three other inviscid amplified $k^{+}$-branches are displayed.

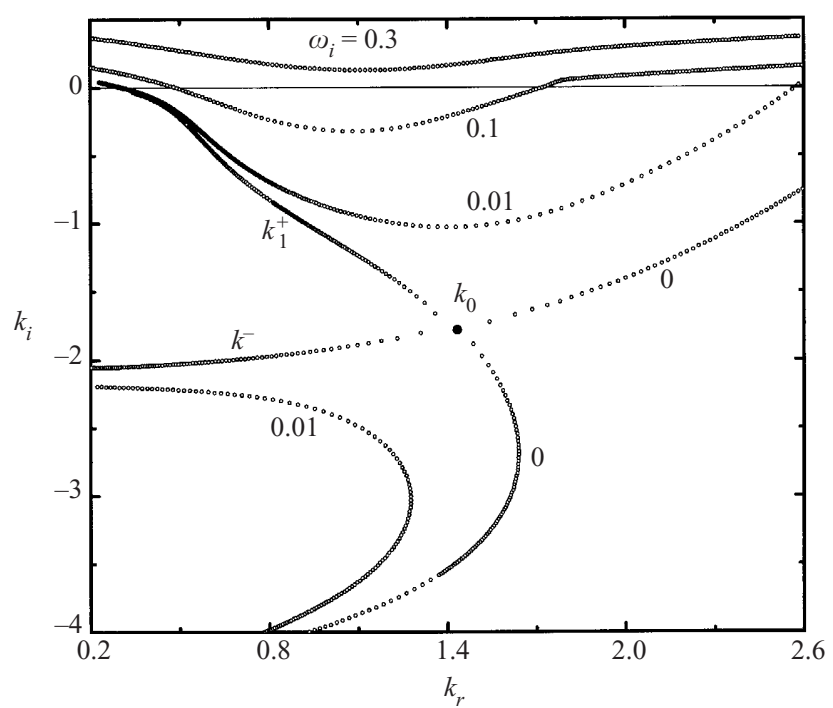

FIGURE 14. Level curves $\omega_{i}=0, \omega_{i}=0.01, \omega_{i}=0.1$ and $\omega_{i}=0.3$ of 'primary' inviscid $k_{1}^{+}$- and $k^{-}$-branches for azimuthal helical mode $m=-3$ in the vicinity of saddle point $k_{0}$ depicted in figure 13 .

convective $(A I-C I)$ transition curve in the $(a, q)$-plane for different Reynolds numbers and azimuthal wavenumbers. An example of transition curve is displayed in figure 15 for $m= \pm 1$ and $R e=100$. The 'jet' side $(a>-0.5)$ and 'wake' side $(a<-0.5)$ of the curve have been obtained by approaching them from the convectively unstable $(C I)$ domains in the $(a, q)$-plane. The closed curves define a pocket of absolute instability $(A I)$ within which the spatial response problem associated with strictly spatial ( $\omega$ real, $k$ complex) branches is ill-posed. The flow is stable $(S)$ outside the swirl 


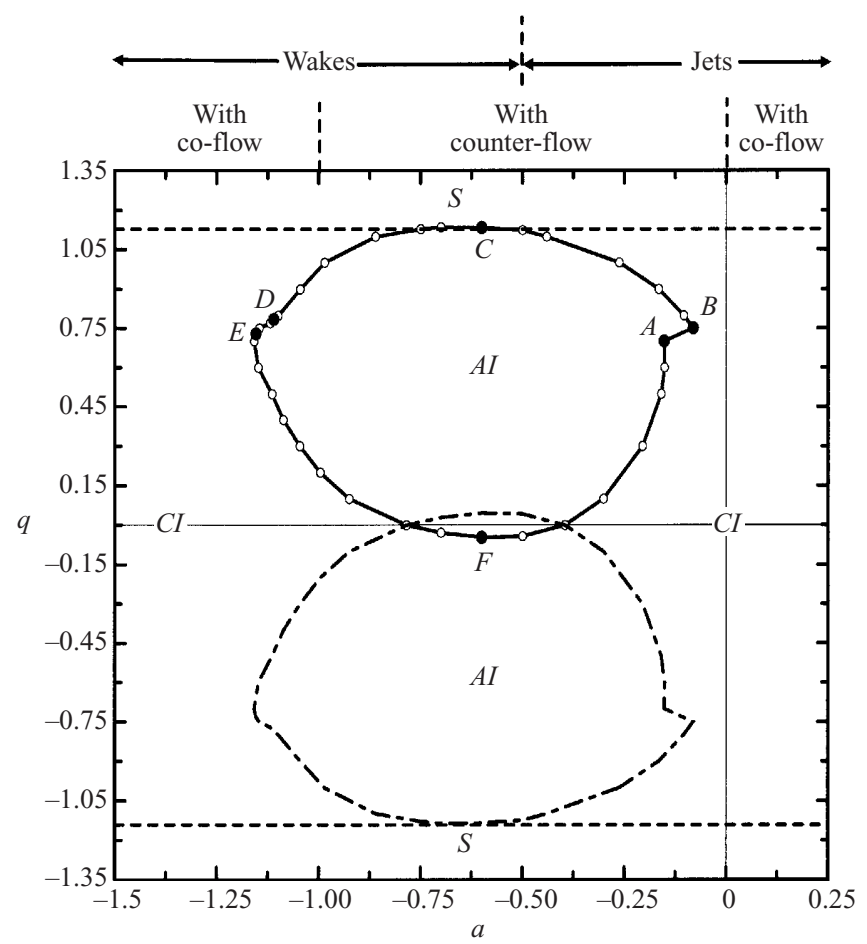

FIGURE 15. Absolute-convective transition curves of inviscid bending modes $m= \pm 1$ in the $(a, q)$-plane at $R e=100$. Domains of stability $(S)$, convective instability $(C I)$ and absolute instability $(A I)$ are indicated. Each symbol $\bigcirc$ corresponds to a numerical computation. The dot-dashed line pertains to the $m=+1$ inviscid mode and is determined by reflection of the $m=-1$ transition curve with respect to the solid horizontal axis of zero swirl.

range corresponding to the two dashed horizontal lines. As expected, note that the transition curves of the $m=-1$ and $m=+1$ modes are symmetric with respect to the horizontal axis. This property holds for all transition curves to be considered in the paper. Let $F$ and $C$ denote the points of marginal stability on the $A I-C I$ transition curve of the $m=-1$ mode. Each open circle refers to a numerical result where a saddle point $k_{0}$ effectively occurs for a real absolute frequency $\omega_{0}$. A complicated sequence of switching events between various inviscid spatial branches takes place as we travel along the $A I-C I$ transition boundary starting from point $F$. Along the path $F A$, the saddle point $k_{0}$ involves pinching between the most amplified $k^{+}$-mode determined in figure 3 and a damped $k^{-}$-mode. Note that the pertinent $k^{+}$-branch then coincides with the most amplified inviscid mode depicted in figure 3 for moderate swirl. A sharp jump to another distinct pinching scenario takes place between $A$ and $B$. More specifically, along the path $B C$ the saddle point becomes associated with a damped $k^{-}$-branch and an amplified hybrid 2-1 $k^{+}$-branch. Conversely, along the path $C D$, the saddle point pertains to an amplified hybrid 2-1 $k^{-}$-branch and a damped $k^{+}$-branch. Another sharp jump takes place between $D$ and $E$. Finally, along the path $E F$, pinching involves an amplified $k^{-}$-branch and a damped $k^{+}$-branch.

The effect of viscosity on the size of the $A I$ bubble is illustrated in figure 16 for the azimuthal mode $m=-1$. For clarity, only the bending mode $m=-1$ is considered. Corresponding results for the $m=+1$ mode may readily be obtained by symmetry as 


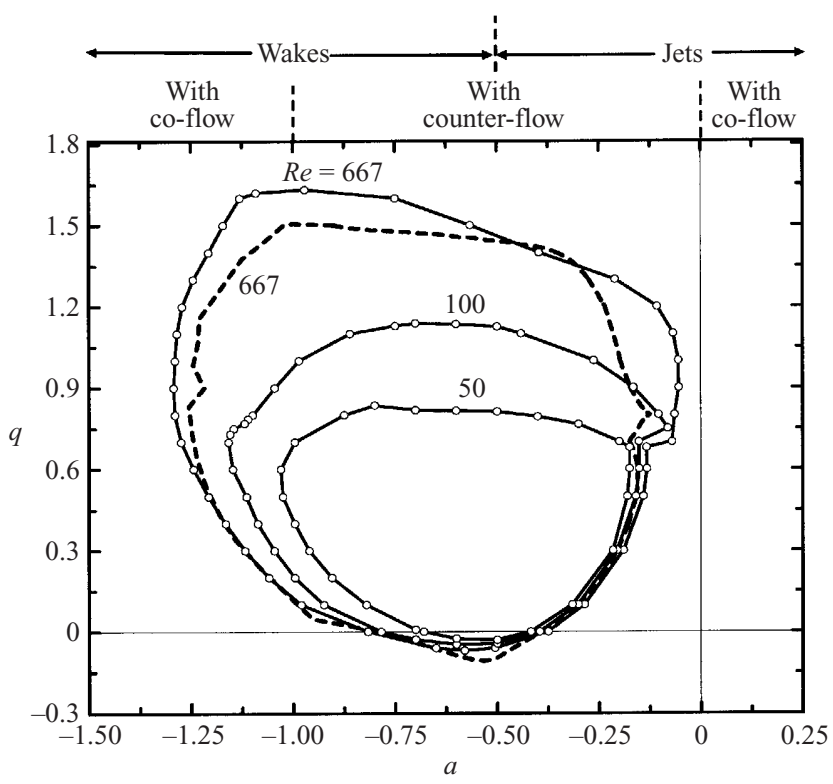

FIGURE 16. Absolute-convective transition curves of inviscid bending mode $m=-1$ in the $(a, q)$-plane at different Reynolds numbers Re. ---, transition boundary obtained by Delbende et al. (1998) at $R e=667$.

in figure 15. Decreasing the Reynolds number dramatically reduces the extent of the AI domain but does not change the nature of the saddle point as described above at $R e=100$. For moderate swirl levels $0<q<0.7$, the sensitivity to changes in $R e$ is weak on the 'jet' side $(a>-0.5)$ and strong on the 'wake' side $(a<-0.5)$. For $q>0.7$, the $A I$ region rapidly shrinks with decreasing $R e$ both on the 'jet' and 'wake' sides. The results of Delbende et al. (1998) at $R e=667$ are represented by a dashed curve. Excellent agreement between the two approaches is obtained for moderate swirl levels. However, significant differences are noteworthy near neutrality, particularly towards high swirls. This feature might be due to numerical diffusion effects which could have enhanced the effective viscosity in the numerical simulations of Delbende et al. (1998). Furthermore, according to the same authors, the numerical spatial discretization error incurred in retrieving the maximum absolute growth rate over all branches is of order $10^{-2}$. Such an error is particularly damaging near the neutral boundary, which could account for the observed differences. Finally, the numerical estimation procedure of Delbende et al. (1998) relies on the fact that the long-time asymptotic regime is effectively reached; a finite-time numerical integration necessarily slightly underestimates absolute growth rates and thereby the size of the AI region. This interpretation is consistent with the present findings: the dashed curve in figure 16 lies within the corresponding $A I$-domain determined by the present approach.

Corresponding results are displayed in figures 17 and 18 for the $m=-2$ and $m=-3$ inviscid azimuthal modes. In contrast with the $m=-1$ case, the same pinching scenario prevails all along the $A I-C I$ transition curves; it always involves the most amplified branch for moderate $q$ and its $k^{-}$or $k^{+}$counterpart. The same qualitative behaviour holds as for the $m=-1$ mode; the $A I$ region shrinks as $R e$ decreases, especially on the 'wake' side or for large swirl. The results of Delbende et al. (1998) at $R e=667$ are also displayed as dashed curves in figures 17 and 18. Both 


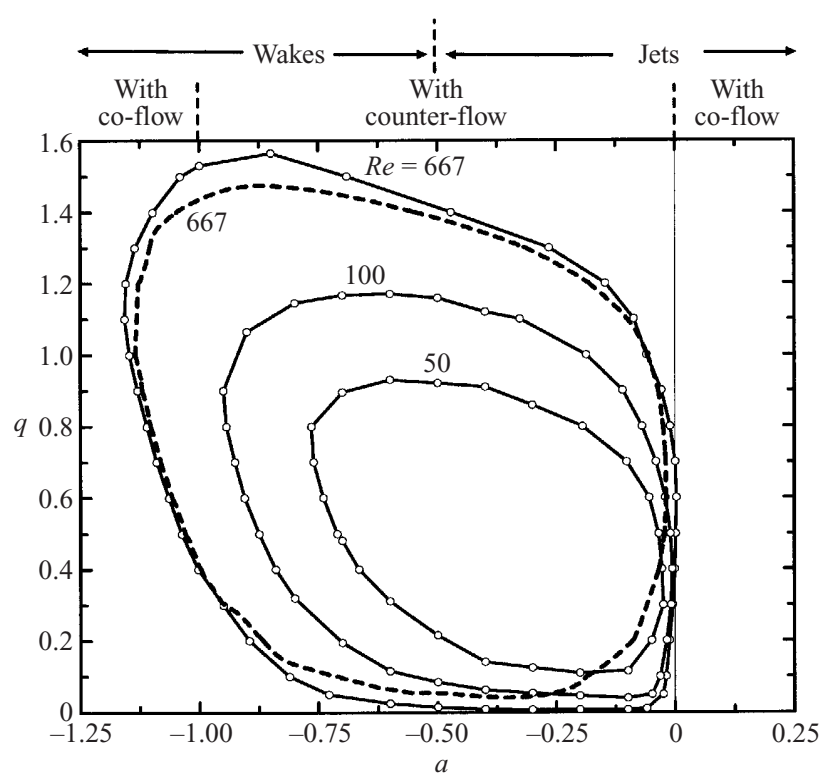

FIGURE 17. Absolute-convective transition curves of inviscid bending mode $m=-2$ in the $(a, q)$-plane at different Reynolds numbers Re.---, transition boundary obtained by Delbende et al. (1998) at $R e=667$.

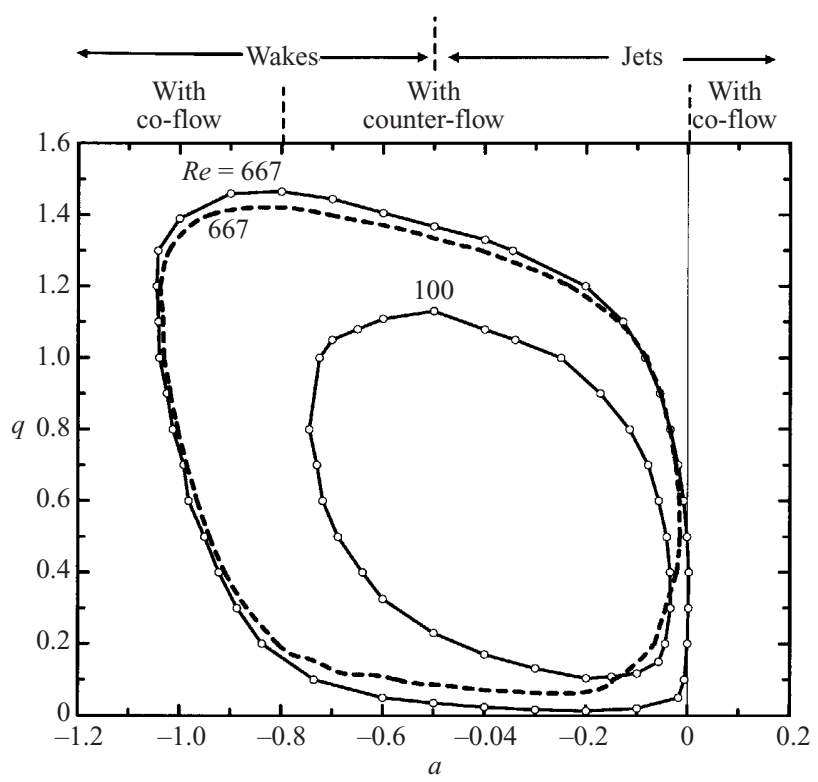

FIGURE 18. Absolute-convective transition curves of inviscid bending mode $m=-3$ in the $(a, q)$-plane at different Reynolds numbers Re. ---, transition boundary obtained by Delbende et al. (1998) at $R e=667$.

analyses yield comparable results. However, significant shifts appear on the 'jet' side for small $q$. An enlarged view of this domain of parameter space is given in figure 22 . In the present investigation, $A I-C I$ transition arises on the 'jet' side for the $m=-2$ and $m=-3$ modes at $R e=667$ and a slightly positive and small co-flow. A small 


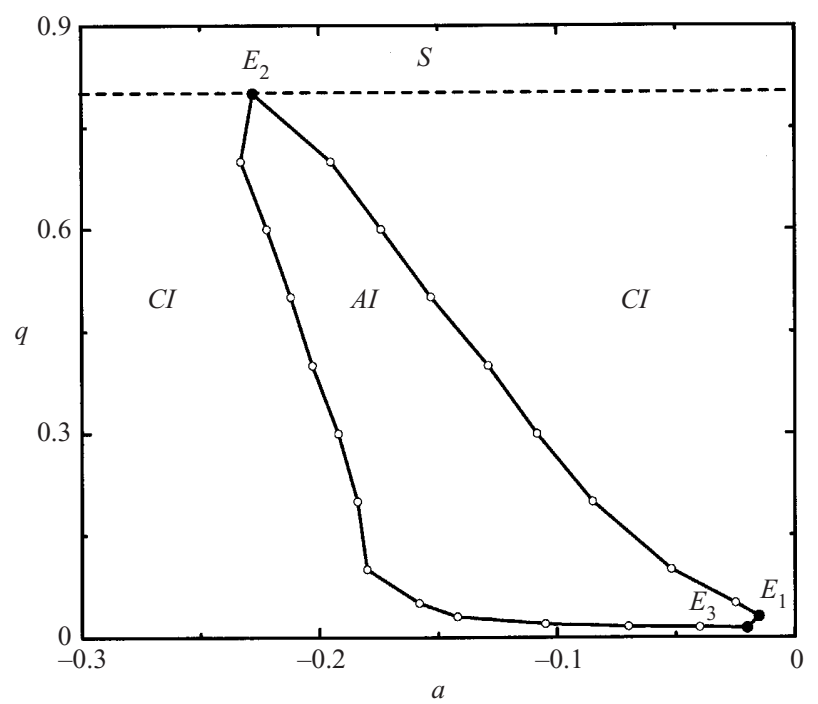

FIGURE 19. Absolute-convective transition curve of viscous bending mode $m=+1$ in the $(a, q)$-plane at $R e=667$.

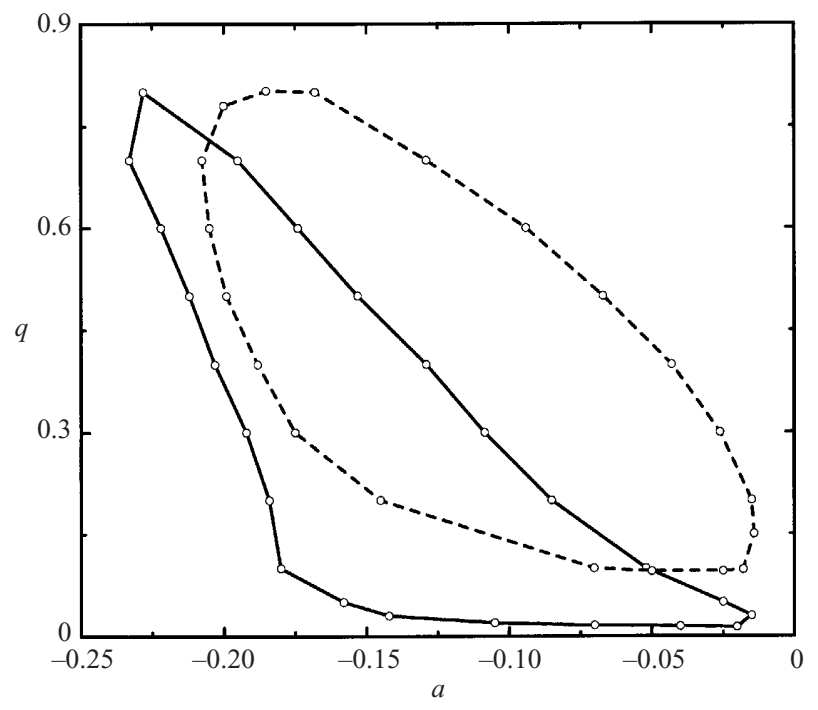

FIGURE 20. Absolute-convective transition curves of viscous bending mode $m=+1$

$$
\text { at }-, R e=667 ;---, R e=100 \text {. }
$$

counterflow ( $a$ slightly negative) was found by Delbende et al. (1998) to be necessary to promote $A I$.

The $A I-C I$ transition curve pertaining to the viscous mode identified in figures 5-7 is displayed in figure 19 for the Reynolds number $R e=667$. In contrast to the case of inviscid modes, its bubble of $A I$ entirely lies on the 'jet' side $(a>-0.5)$. Its boundary is associated with pinching of the same $k^{+}$-branch with distinct $k^{-}$-branches on the segments $E_{1} E_{2}$ and $E_{2} E_{3}$. The effects of Reynolds number on the extent of the $A I$ pocket is depicted in figure 20; as $R e$ decreases below 667 to $R e=100$, the $A I$ domain is seen to widen and to shift further towards the co-flow jet side. An increase in Re 


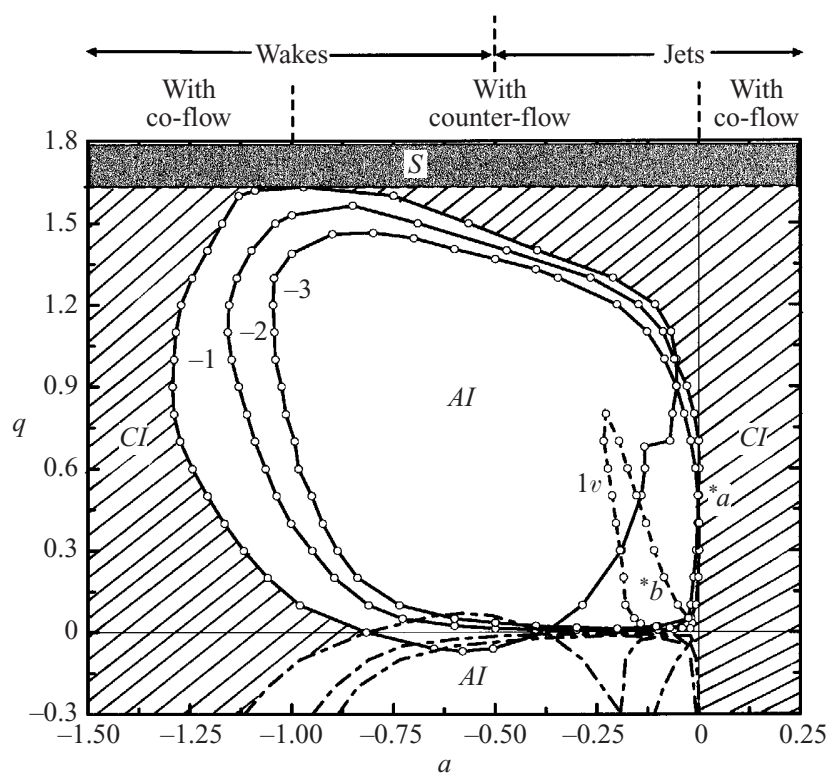

FIGURE 21. Overall absolute-convective transition boundary of Batchelor vortex in the $(a, q)$-plane at $R e=667$. Solid lines, labelled with the associated azimuthal wavenumber $m$, delineate both inviscid and viscous pockets of absolute instability pertaining to helical modes $m= \pm 1, m= \pm 2$ and $m= \pm 3$. Hatched and dark grey areas indicate domains of convective instability and stability, respectively. The bending $(m=+1)$ viscous mode is indicated by the symbol $1 v$.

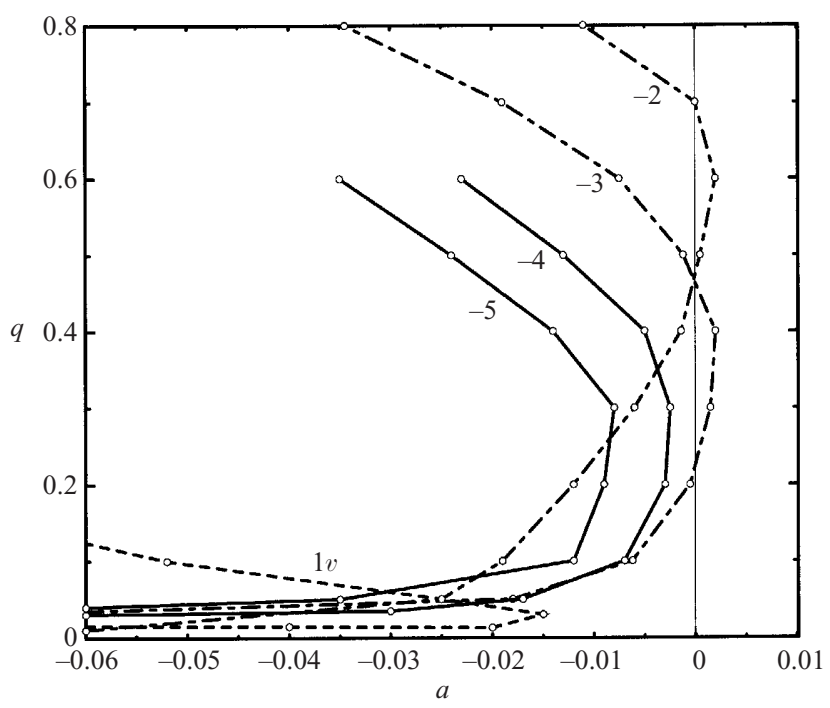

FiguRE 22. Magnification, on the 'jet' side and for small swirl values, of the absolute-convective transition curves in figure 21 for inviscid modes $m=-2,-3,-4$ and -5 and viscous mode $m=+1$ at $R e=667$. The azimuthal wavenumber is indicated next to each curve.

above 667 would result in the collapse of the $A I$ region, in full agreement with the viscous nature of this mode.

Overlaying the $A I-C I$ transition curves for various helical modes $m= \pm 1, \pm 2, \pm 3$ leads to the determination of the overall $A I-C I$ transition boundary in the $(a, q)$-plane 


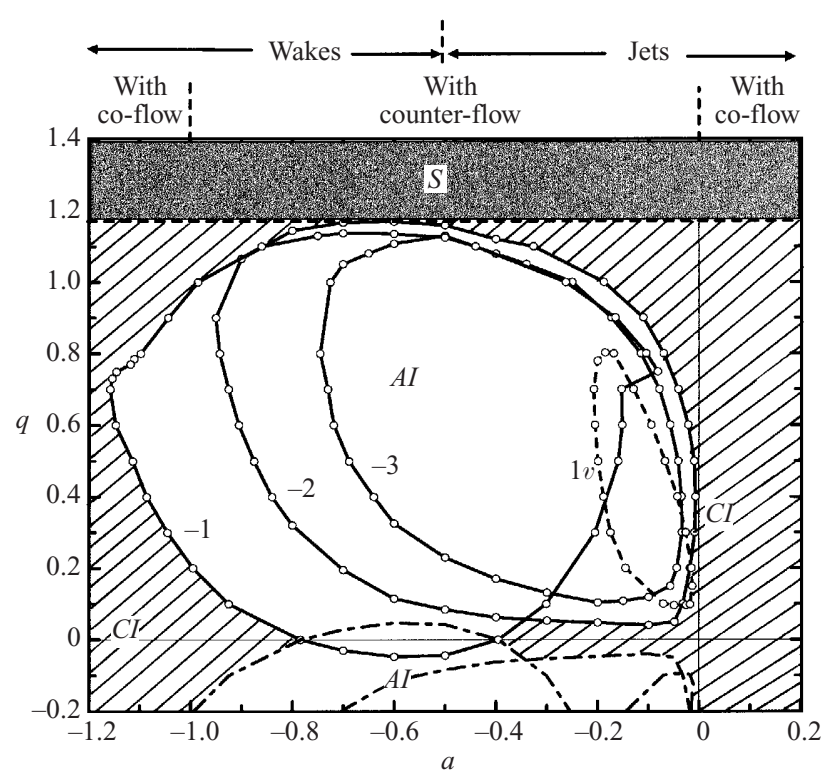

FIgURE 23. Counterpart of figure 21 at $R e=100$.

at $R e=667$ as shown in figure 21. Such a state diagram constitutes the main result of the present investigation. The hatched and dark grey areas, respectively, indicate the $C I$ and $S$ regions of parameter space. The extent of the $A I$ region (clear area) is approximatively the same as in Delbende et al. (1998). The $A I$ range of the external flow parameter widens as $q$ increases from zero to moderate values $(0<q<0.7)$ and shrinks for higher levels as the stability boundary is approached. Furthermore, $A I-C I$ transition is triggered by distinct inviscid or viscous azimuthal modes; on the 'wake' side $(a<-0.5)$, the bending mode $m=-1$ is always critical whereas on the 'jet' side $(a>-0.5)$ various helical modes partake in defining the transition curve, as illustrated by the magnified plot of figure 22 . For very low $q$, in the range $0 \leqslant q<0.015$, the inviscid mode $m=-2$ is critical, whereas in the interval $0.015<q<0.044$, it is superseded by the viscous mode $m=-1$. For higher swirl levels, in the ranges $0.044<q<0.078,0.078<q<0.474,0.474<q<0.983, q>0.983$, transitions successively take place via the inviscid modes $m=-4,-3,-2,-1$. In contrast with the finding of Delbende et al. (1998), the helical mode $m=-5$ is never critical at $R e=667$. For zero external flow jets at $a=0$, absolute instability first sets in via the helical mode $m=-3$ at $q=0.225$. A second helical mode $m=-2$ becomes $A I$ as $q$ exceeds the value 0.467 . Beyond $q=0.7$, the zero external flow jet returns to $C I$.

The effect of increasing viscosity is illustrated by comparing the $A I$ domains of figures 21 and 23 at $R e=667$ and $R e=100$, respectively. The hierarchy of helical modes is preserved on the 'wake' side provided that $a<-0.86$. When $a>-0.86$, on the high $q$ 'wake' side, the critical mode switches from $m=-1$ to $m=-2$. On the 'jet' side, the hierarchy is significantly modified; the inviscid mode $m=-1$ is critical in the interval $0 \leqslant q<0.057$ whereas the inviscid mode $m=-2$ takes over in the disjoint intervals $0.057<q<0.096$ and $0.096<q<1.2$. Finally, $C I-A I$ transition takes place via the viscous mode $m=+1$ in the range $0.057<q<0.096$. Contrary to the case $R e=667$, zero external flow jets remain $C I$ at all swirl levels $q$.

The effect of vanishing viscosity is depicted in figure 24 at $R e=10000$ for the inviscid modes $m=-1$ and $m=-2$ only. The strictly inviscid results of Olendraru 


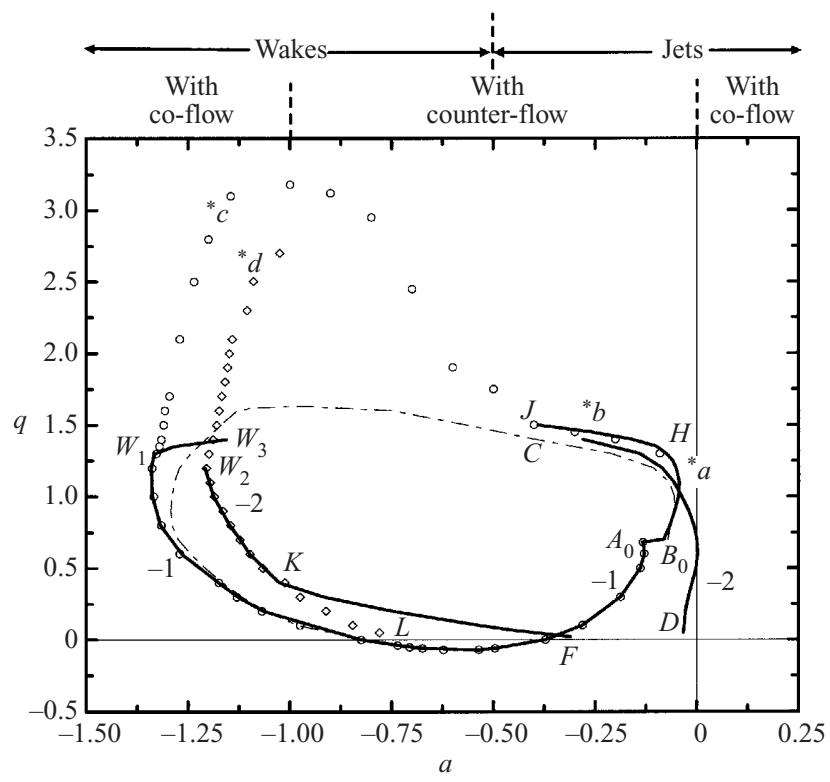

FIGURE 24. 'Inviscid' absolute-convective transition curves in the $(a, q)$-plane. Present spectral method at $R e=10000$ ( $O, m=-1 ; \diamond, m=-2)$. Shooting method at $R e=\infty$ of Olendraru et al. (1996, 1999) (solid lines). Present spectral method at $R e=667$ (dashed line $m=-1$ ). Star symbols refer to parameter settings selected for the representation of eigenfunctions in figure 12 . Note that the scale of the figure is smaller than in other comparable figures 21 and 23.

et al. $(1996,1999)$ are indicated by solid curves. The inviscid results agree perfectly with those of the present viscous analysis except along the solid segments $A_{0} H, C D$ on the jet side, $W_{1} W_{3}$ on the wake side and $K F$ in the counter flow domain. On the jet side, the viscous code fails in providing the transition curves $C D$ for the azimuthal wavenumber mode $m=-2$ and $A_{0} H$ for the helical mode $m=-1$. The inviscid results of Olendraru et al. $(1996,1999)$ fill this gap. According to the inviscid study, the critical point in this region lies close to the real $r$-axis and the number of collocation points selected in the viscous spectral code is insufficient to resolve the critical-layer structure. On the wake side, the viscous transition curve of the $m=-1$ mode bifurcates away from the solid curve of Olendraru et al. (1999) beyond the point $W_{1}$. This is because the shooting method implemented in the inviscid study of Olendraru et al. (1999) tracks the same pinching downstream $k^{-}$-branch. The spectral method used here in a viscous context reveals that another downstream $k^{-}$-branch is also involved in a pinching process which takes over beyond $W_{1}$. A bifurcation of a different nature takes place in the vicinity of $K$ for the $m=-2$ mode; this time, the shooting method of Olendraru et al. $(1996,1999)$ has jumped from one $k^{-}$-mode to another, since many nearly neutral modes are observed to cluster, as in Leibovich \& Stewartson (1983). The spectral method indicates that the same $k^{-}$-mode should be tracked in order to obtain the correct absolute-convective transition boundary $K L$. The boundary of the absolutely unstable region is seen to extend far above the value $q \sim 1.5$ previously obtained by Delbende et al. (1998) at $R e=667$. At $R e=10000$, the Batchelor vortex only becomes stable with respect to the $m=-2$ and $m=-1$ modes above $q \sim 3.1$ corresponding to point $S$ in figure 24. As the Reynolds number increases from $R e=100$ (figure 23) through $R e=667$ (figure 23) to $R e=10000$ (figure 24). The absolutely unstable region successively extends to larger swirl values 
$q=1.2,1.6$ and 3.1 beyond which the Batchelor vortex becomes linearly stable. Along the segment $W_{1} S$, the same upstream $k^{+}$-branch is involved in the pinching process but the pinching downstream $k^{-}$-branch changes at discrete points as we proceed from $W_{1}$ to $S$. It is observed that the selected downstream $k^{-}$-branch is always the most spatially amplified. The same scenario prevails along the segment $S J$, provided that the roles of $k^{+}$and $k^{-}$are switched. Furthermore, over the entire curve $W_{1} S J$, the spatial growth rate $\pm \operatorname{Im}\left(k_{0}\right)$ is found to be very small so that the associated mode is nearly neutral. As discussed below, the corresponding eigenfunctions are, in fact, the near-neutral centre-modes first theoretically predicted by Stewartson \& Brown (1985) within a temporal inviscid framework, in specific swirl parameter ranges. This has been checked by continuously changing the spatial branches ( $k$ complex, $\omega$ real) into their temporal counterparts ( $k$ real, $\omega$ complex) (Olendraru et al. 1999). This appears to be the first numerical evidence for the existence of such a class of near-neutral perturbations. We may wonder whether this upper swirl value reaches a finite limit as the Reynolds number goes to infinity. Note that, as $q \rightarrow \infty$, the Batchelor vortex reduces to a pure vortex (without axial velocity) with a strictly positive radial gradient of axial vorticity. Under such conditions (Ash \& Khorrami 1995), the basic flow is inviscidly stable as $q \rightarrow \infty$. According to this argument, it can therefore be concluded that, as the Reynolds number tends to infinity, the absolutely unstable domain is confined within a band of finite swirl parameters.

\section{Conclusions}

The present investigation has focused on the effects of viscosity on the spatial instability properties and absolute-convective transitions in the Batchelor vortex. The main results are outlined below.

The detailed spatial instability characteristics have been documented in a wide range of Reynolds numbers $(100<R e<10000)$ for the case of a zero co-flow jet $(a=0)$. At moderate swirl levels ( $q$ of order unity or less), 'inviscid' spatial modes quickly reach their asymptotic limit provided the Reynolds number exceeds $R e=2000$, which may be regarded as effectively infinite. At large swirl levels $(q$ larger than unity), the growth rates of inviscid spatial modes are weak and highly sensitive to the magnitude of the Reynolds number, as evidenced by the rapid changes in the absolute-transition instability boundary. These nearly neutral modes are of the same variety as the centre-modes theoretically predicted by Stewartson \& Brown (1985). For intermediate Reynolds numbers, 'viscous' bending $(m= \pm 1)$ modes have been identified, which are the spatial counterparts of the temporal asymmetric modes discovered by Khorrami (1991) and further confirmed by Mayer \& Powell (1992). Note that we have also determined a viscous spatial axisymmetric mode $(m=0)$, as in the previously mentioned temporal analyses, but the results have not been presented here. Whether or not this axisymmetric viscous mode becomes critical remains an unresolved issue. Hybrid modes have also been encountered as in the temporal case (Khorrami 1991).

The effect of the Reynolds number on the absolute-convective instability transition curves has been thoroughly analysed. At moderate swirl levels ( $q$ of order unity or less), transition curves on the jet side are fairly insensitive to a decrease in Reynolds number, whereas, on the wake side, the absolutely unstable domain rapidly shrinks (figures 16 and 17) with decreasing $R e$. Furthermore, jets with a slight co-flow $(a \geqslant 0)$ may become absolutely unstable if the Reynolds number is large enough (figure 22). At large swirl levels ( $q$ larger than unity), the transition curve becomes very sensitive 
to the Reynolds number (figures 16, 17 and 24): the absolute-convective boundary shifts to higher swirls, far above unity, with increasing Reynolds number. Viscous bending modes $(m= \pm 1)$ contribute to the determination of the overall transition curve on the jet side (figures 21-23) for low $q$, in an intermediate range of Reynolds numbers.

As a final word of caution, we should emphasize that no definite connection has yet been established between the present absolute/convective local instability analysis and the occurrence of axisymmetric vortex breakdown. By contrast, the existence of a global axisymmetric mode has been demonstrated by Wang \& Rusak (1996) and Ruzak, Wang \& Whiting (1998) for vortex flows with co-flow jets of sufficiently large swirl. Such a mode is likely to govern the transition to axisymmetric vortex breakdown as convincingly argued by Wang \& Ruzak (1997). However, it appears plausible that the unsteady wake structure dowstream of the vortex breakdown results from a helical global mode induced by a transition from convective to absolute instability.

The authors are indebted to Professor P. Huerre, Drs I. Delbende and T. Loiseleux for fruitful discusssions and they express their warm thanks to Professor Huerre for his generous help in revising the manuscript. The authors acknowledge financial support by the Direction des Recherches, Etudes et Techniques (DRET) of the French Ministry of Defense under grant number 92-098. Computational facilities have been provided by the Institut du Développement et des Ressources en Informatique Scientifique (IDRIS/CNRS).

\section{REFERENCES}

Ash, R. L. \& Khorrami, M. R. 1995 Vortex stability. In Fluid Vortices (ed. S. I. Green), pp. 317-372. Kluwer.

Batchelor, G. K. 1964 Axial flow in trailing line vortices. J. Fluid Mech. 20, 645-658.

BAtChelor, G. K. \& Gill, A. E. 1962 Analysis of the stability of axisymmetric jets. J. Fluid Mech. 14, 529-551.

Benjamin, T. B. 1962 Theory of the vortex breakdown. J. Fluid Mech. 14, 593-629.

BERS, A. 1983 Space-time evolution of plasma instabilities-absolute and convective. In Handbook of Plasma Physics (ed. M. N. Rosenbluth \& R. Z. Sagdeev), vol. 1, pp. 451-517. North-Holland.

BRIGGS, R. J. 1964 Electron-Stream Interaction with Plasmas. MIT Press.

Cotton, F. W. \& Salwen, H. 1981 Linear stability of rotating Hagen-Poiseuille flow. J. Fluid Mech. 108, 101-125.

Delbende, I., Chomaz, J. M. \& Huerre, P. 1998 Absolute/convective instabilities in the Batchelor vortex: a numerical study of the linear impulse response. J. Fluid Mech. 355, 229-254.

Delery, J. M. 1990 Aspects of vortex breakdown. Prog. Aerospace Sci. 30, 1-59.

Duck, P. W. \& Foster, M. R. 1980 The inviscid stability of a trailing line vortex. Z. angew. Math. Phys. 31, 523-530

Duck, P. W. \& Khorrami, M. R. 1992 A note on the effects of viscosity on the stability of a trailing-line vortex. J. Fluid Mech. 245, 175-189.

Faler, J. H. \& Leibovich, S. 1977 Disrupted states of vortex flow and vortex breakdown. Phys. Fluids A 20, 1385-1400.

Garg, A. K. \& Leibovich, S. 1979 Spectral characteristics of vortex breakdown flowfields. Phys. Fluids A 22, 2053-2064.

Gottlieb, D. \& Orszag, A. 1977 Numerical Analysis of Spectral Methods: Theory and Applications. SIAM, Philadelphia.

Huerre, P. 2000 Open shear flow instabilities. In Perspectives in Fluid Dynamics (ed. G. K. Batchelor, H. K. Moffatt \& M. G. Worster), pp. 159-229. Cambridge University Press.

Huerre, P. \& Monkewitz, P. A. 1985 Absolute and convective instabilities in free shear layers. J. Fluid Mech. 145, 151-168. 
Huerre, P. \& Monkewitz, P. A. 1990 Local and global instabilities in spatially developing flows. Annu. Rev. Fluid Mech. 22, 473-537.

Huerre, P. \& Rossi, M. 1998 Hydrodynamic instabilities in open flows. In Hydrodynamics and Nonlinear Instabilities (ed. C. Godreche \& P. Manneville), pp. 81-294. Cambridge University Press.

Khorrami, M. R. 1991 On the viscous modes of instability of a trailing line vortex. J. Fluid Mech. 255, 197-212.

Khorrami, M. R. 1992 Behaviour of asymmetric unstable modes of a trailing line vortex near the upper neutral curve. Phys. Fluids A 4, 1310-1313.

Khorrami, M. R., Malik, M. R. \& Ash, R. L. 1989 Applications of spectral collocations techniques to the stability of swirling flows. J. Comput. Phys. 81, 206-229.

Leibovich, S. 1978 The structure of vortex breakdown. Annu. Rev. Fluid Mech. 10, 221-246.

LeIBOVICH, S. 1983 Vortex stability and breakdown: survey and extension, AIAA J. 22, 1192-1206.

Leibovich, S. \& Stewartson, K. 1983 A sufficient condition for the instability of columnar vortices. J. Fluid Mech. 126, 335-356.

Lessen, M. \& Paillet, F. 1974 The stability of a trailing line vortex. Part 2. Viscous theory. J. Fluid Mech. 65, 769-779.

Lessen, M. \& Singh, P. J. 1974 The stability of axisymetric free shear layers. J. Fluid Mech. 60, 433-457.

Lessen, M., Singh, P. \& Paillet, J. F. 1974 The stability of a trailing line vortex. Part 1. Inviscid theory J. Fluid Mech. 63, 753-763.

Loiseleux, T., Chomaz. J. M. \& Huerre, P. 1998 The effect of swirl on jets and wakes: linear instability of the Rankine vortex with axial flow. Phys. Fluids 10, 1120-1134.

Loiseleux, T., Delbende I. \& Huerre, P. 2000 Absolute and convective instabilities of a swirling jet/wake shear layer. Phys. Fluids 12, 375-380.

Malik, M. R., Zang, T. A. \& Hussaini, M. Y. 1985 A spectral collocation method for the Navier-Stokes equations. J. Comput. Phys. 61, 64-88.

Mayer, E. W. \& Powell, K. G. 1992 Viscous and inviscid instabilities of a trailing line vortex. J. Fluid Mech. 245, 91-114.

Olendraru, C., Sellier, A. \& Huerre, P. 1999 Inviscid instability of the Batchelor vortex: absolute-convective transition and spatial branches. Phys. Fluids 11, 1805-1820.

Olendraru, C., Sellier, A., Rossi, M. \& Huerre, P. 1996 Absolute-convective instability of the Batchelor vortex. C. R. Acad. Sci. Paris Sér. II b, 323, 153-159.

RuZAK, Z., WANG, S. \& Whiting, C. H. 1998 The evolution of a perturbed vortex in a pipe to axisymmetric vortex breakdown. J. Fluid Mech. 366, 211-237.

StewARTSON, K. \& BRown, S. N. 1985 Near-neutral centre-modes as inviscid perturbations to a trailing line vortex. J. Fluid Mech. 156, 387-399.

Stewartson, K. \& CAPEll, K. 1985 On the stability of ring modes in a trailing line vortex: the upper neutral points. 156, 369-386.

TsaI, C.-Y. \& WidnalL, S. E. 1980 Examination of a group-velocity criterion for breakdown of vortex flow in a divergent duct. Phys. Fluids A 23, 864-870.

WANG, S. \& RuZAK, Z. 1996 On the stability of an axisymmetric rotating flow in a pipe. Phys. Fluids 8, 1007-1016.

WANG, S. \& RUZAK, Z. 1997 The dynamics of a swirling flow in a pipe and transition to axisymmetric vortex breakdown. J. Fluid Mech. 340, 177-223. 